Suárez-Rico, Y., Gómez-Villegas, M., \& GarcíaBenau, M. (2021). La utilidad de los informes de sostenibilidad en entidades públicas y privadas en Colombia: la percepción de los stakeholders. Contaduría Universidad de Antioquia, 79, 103-136. Doi: https://doi.org/10.17533/udea.rc.n79a05

\title{
La utilidad de los informes de sostenibilidad en entidades públicas y privadas en Colombia: la percepción de los stakeholders
}

Yuli Marcela Suárez-Rico

Universidad Nacional de Colombia ymsuarezr@unal.edu.co

Orcid: 0000-0002-3754-6600

Mauricio Gómez-Villegas Universidad Nacional de Colombia mgomezv@unal.edu.co

Orcid: 0000-0001-9043-6358

María Antonia García-Benau

Universidad de Valencia

Maria.garcia-benau@uv.es

Orcid: 0000-0002-9331-9103 
La utilidad de los informes de sostenibilidad en entidades públicas y privadas en Colombia: la percepción de los stakeholders

Resumen: El aumento en la divulgación de información social y medioambiental (de sostenibilidad) genera preguntas sobre su utilidad y materialidad, lo que implica conocer las expectativas de los stakeholders. El stakeholder engagement permite señalar que la participación de los grupos de interés no solo debe centrarse en la producción de la información en las empresas, sino también en la definición de los marcos y estándares que estructuran estos reportes. Este trabajo busca caracterizar las expectativas y la percepción que tienen diversos stakeholders, sobre la utilidad de los informes de sostenibilidad emitidos por organizaciones públicas y privadas en Colombia. A partir de dos cuestionarios respondidos por 62 gestores, preparadores, auditores, reguladores, estudiantes y profesores, se identifican temas que los marcos más extendidos (GRI) no incluyen y que incrementarían la materialidad de esta información en Colombia: acciones para la paz, lucha contra la corrupción y la desigualdad, información para los ciudadanos, entre otros.

Palabras Clave: Materialidad; expectativas; GRI; información social y ambiental: stakeholer engagement.

Usefulness of sustainability reporting in Colombian public and private entities: stakeholders' perceptions

Abstract: Increased disclosure of social and environmental (sustainability) information raises questions about its usefulness and materiality, which implies knowing the expectations of stakeholders. Stakeholder engagement allows to point out that stakeholder participation should not only focus on the production of information in companies, but also on the definition of the frameworks and standards that structure these reports. Based on two questionnaires answered by 62 managers, preparers, auditors, regulators, students and teachers, we identified topics that the most extended frameworks (GRI) do not include and that would increase the materiality of this information in Colombia: actions for peace, fight against corruption and inequality, information for citizens, among others.

Keywords: Materiality; expectations; GRI; social and environmental information; stakeholder engagement.

A utilidade dos relatórios de sustentabilidade em entidades públicas e privadas na Colômbia: A percepção dos stakeholders

Resumo: $O$ aumento na divulgação de informação social e do meio ambiente (de sustentabilidade) gera perguntas sobre sua utilidade e materialidade, o que implica conhecer as expectativas dos stakeholders. $O$ engajamento dos stakeholders permite evidenciar que a participação dos grupos de interesse não só deve se centrar na produção da informação nas empresas, mas também na definição dos marcos e estândares que estruturam estes relatórios. Este trabalho busca caracterizar as expectativas e a percepção que têm diversos stakeholders, sobre a utilidade dos relatórios de sustentabilidade emitidos por organizações públicas e privadas na Colômbia. A partir de dois questionários respondidos por 62 gestores, preparadores, auditores, reguladores, estudantes e professores, se identificam assuntos que os marcos mais estendidos (GRI) não incluem e que incrementariam a materialidade desta informação na Colômbia: ações para a paz, luta contra a corrupção e a desigualdade, informação para os cidadãos, entre outros.

Palavras chave: Materialidade, expectativas; GRI, informação social e do meio ambiente: engajamento dos stakeholders.

L'utilité des rapports de durabilité dans les entités publiques et privées en Colombie : les perceptions des stakeholders

Résumé: L'augmentation de la divulgation d'informations sociales et environnementales (de durabilité) soulève des questions sur leur utilité et leur importance relative, ce qui implique de connaître les attentes des stakeholders. Le stakeholder engagement permet de souligner que la participation des parties prenantes ne doit pas seulement se concentrer sur la production d'informations dans les entreprises, mais aussi sur la définition des cadres et des normes qui structurent ces rapports. Cet article cherche à caractériser les attentes et les perceptions des différentes parties prenantes concernant l'utilité des rapports de durabilité publiés par les organisations publiques et privées en Colombie. Sur la base de deux questionnaires auxquels ont répondu 62 gestionnaires, préparateurs, auditeurs, régulateurs, étudiants et professeurs, nous identifions les points que les cadres les plus répandus (GRI) n'incluent pas et qui augmenteraient la matérialité de ces informations en Colombie : actions pour la paix, lutte contre la corruption et les inégalités, information des citoyens, entre autres.

Mots clés: importance relative, attentes, GRI, rapports sociaux et environnementaux, stakeholder engagement. 
Cont. udea (julio-septiembre, pp. 103-136. (c) Universidad de Antioquia-2021.

\title{
La utilidad de los informes de sostenibilidad en entidades públicas y privadas en Colombia: la percepción de los stakeholders
}

\author{
Yuli Marcela Suárez-Rico, Mauricio Gómez-Villegas y María Antonia García-Benau \\ https://doi.org/10.17533/udea.rc.n79a05
}

Primera versión recibida en marzo de 2021 - Versión aceptada en agosto de 2021

\section{Introducción}

La creciente divulgación de información social y medioambiental (de sostenibilidad), en los sectores público y privado, se presenta como un medio para mejorar la rendición de cuentas (accountability) y la transparencia de las organizaciones, como respuesta a las presiones sociales y de mercado que reclaman una mejor gobernanza y un comportamiento socialmente responsable por parte de gobiernos y corporaciones (Kolk, 2008; García-Sánchez, 2021). En este contexto, Colombia es uno de los países pioneros en la producción de información corporativa de sostenibilidad, con base en los lineamientos del Global Reporting Initiative (GRI) (Ariza-Buenaventura, 2012; Gómez y Quintanilla, 2012), siendo el segundo país de la región en reportes de sostenibilidad en 2016, después de Brasil y situándose internacionalmente por encima de España, Italia y Holanda (GRI, 2019b).

Varias empresas colombianas, como Bancolombia o Nutresa, son consideradas pioneras y líderes en la divulgación de esta información, figurando en índices como el Dow Jones Sustainability Index, el DJSI Mila Pacific Alliance y el FTSE4Good (Portafolio, 2018). La expansión de este tipo de información en Colombia ha implicado que entidades públicas, tales como universidades, agencias de gobierno y empresas estatales, también divulguen informes sociales y medioambientales (Flórez-Parra, López-Pérez y López-Hernández, 2017; GómezVillegas, 2017). Tal es el caso de EPM, CHEC, Ecopetrol, ETB, ISA e ISAGEN.

Los reportes de sostenibilidad son preparados atendiendo a diversas guías o estándares, que reclaman la participación de los grupos de interés — stakeholder engagement - con el fin de incluir la información relevante que pueda 
La utilidad de los informes de sostenibilidad en entidades públicas y privadas en Colombia...

influir en sus decisiones (AccountAbility, 2008; GRI, 2014). Tales guías y estándares son explícitos en mencionar que las organizaciones son las encargadas de identificar sus stakeholders y los asuntos materiales a tratar en sus informes.

Manetti (2011) y Moratis y Brandt (2017), han planteado que los procesos de consulta que las corporaciones realizan con sus stakeholders rara vez implican una preocupación genuina por atender sus necesidades y expectativas, siendo utilizadas en mayor medida como una forma de manipulación de las expectativas (Manetti, 2011), o como un intento de construcción de reputación (Bebbington, Larrinaga y Moneva, 2008).

Desde el punto de vista estratégico (Freeman, 1999), las expectativas y las necesidades de los stakeholders son definidas, gestionadas y abordadas en los informes de sostenibilidad desde la visión organizacional y, mayoritariamente, gerencial (Manetti, 2011); esto podría limitar tanto la participación de aquellos grupos que cuentan con menos capacidad de influir en la toma de decisiones corporativa, como la capacidad para mejorar la accountability (Hood, 2007; 2010). Asimismo, el proceso de elaboración de marcos y estándares de información de sostenibilidad reclama la participación de diversos stakeholders, para conseguir un tipo de vinculación efectivo que haga material y útil la información contenida en los reportes. Con ello, el stakeholder engagement implica que la participación de los grupos de interés no solo debe centrarse en la producción de la información en las empresas, sino también en la definición de los marcos y estándares que estructuran estos reportes (Grushina, 2017). Existe poca literatura que explore las expectativas y percepciones de los stakeholders frente a la utilidad de la información de sostenibilidad (García-Sánchez, 2021), lo que también es un vacío en el contexto colombiano. Esto justifica la necesidad de indagar por las expectativas de diversos stakeholders sobre la utilidad y la materialidad de los informes sociales y medioambientales.

Así, el objetivo de este artículo es caracterizar las expectativas y la percepción que tienen diversos stakeholders, sobre la utilidad de los informes de sostenibilidad emitidos por organizaciones públicas y privadas en Colombia. En este contexto, este artículo pretende abordar los siguientes interrogantes: ¿Cuáles son las expectativas de los stakeholders en la información social y ambiental de las organizaciones colombianas? y ¿Cuál es la utilidad percibida por los stakeholders sobre la información social y ambiental en Colombia?

Este estudio contribuye en la caracterización y comprensión de las necesidades y las expectativas de diversos grupos de interés desde una mirada alternativa a la planteada por el enfoque de gestión estratégica de stakeholders, a la vez que permite poner en tensión la concepción banalizada ${ }^{1}$ de utilidad, razonabilidad

1 Banal en el sentido de que son generalizadas pero poco examinadas, tal como ha sido señalado por O’Neill (2002) y Hood (2007) para las concepciones de Accountability y Transparencia. 
de las expectativas e intereses, y accountability (O'neill, 2002; Hood, 2010). De otra parte, este trabajo arroja luces para los emisores de estándares en materia de información social y ambiental, con el propósito de que contemplen una perspectiva amplia sobre la utilidad y las expectativas de diversos stakeholders, identificando temas que no están incorporados en las guías o estándares de mayor utilización (GRI), pero que podrían tener relevancia contextual para los grupos de interés en Colombia.

El documento se estructura en seis secciones adicionales a la presente introducción. La segunda sección aborda las definiciones y perspectivas del stakeholder engagement. La tercera sección realiza una evaluación crítica a la metodología del GRI para la definición de expectativas de los stakeholders frente a la divulgación de información social y ambiental. La cuarta sección expone la metodología empleada en el estudio. En la quinta sección se presentan y discuten los resultados de la encuesta y en la sexta sección se presentan las conclusiones.

\section{Stakeholder Engagement: definiciones y perspectivas}

La noción tradicional del management, economicista y enfocada en satisfacer prioritariamente a los accionistas (Friedman, 1970), está siendo reevaluada, reconociendo la importancia de diversos stakeholders que afectan o son afectados por la organización (Freeman, 1984, 1999). En consecuencia, en los últimos años la gestión ha involucrado una perspectiva que busca definir operativamente qué actores cuentan para la organización y de qué forma existen restricciones para atender todas sus demandas de forma simultánea, en función de diversos atributos como el poder, la legitimidad y la urgencia (Mitchell, Agle y Wood, 1997).

La literatura sobre la información socioambiental plantea que el diálogo con los grupos de interés es la manera más directa para conocer los aspectos económicos, sociales y ambientales, relevantes para los stakeholders, que las organizaciones deberían comunicar en sus informes (Rinaldi, 2013). Thomson y Bebbington (2005) definieron el stakeholder engagement como el rango de prácticas en que las organizaciones toman un enfoque estructurado para consultar a los stakeholders potenciales. Manetti (2011), alude al stakeholder engagement como un proceso de relacionamiento en el que las organizaciones intentan gestionar las expectativas con un balance de intereses y posiciones y en el que se involucra a los grupos de interés en la toma de decisiones, permitiéndoles compartir información, dialogar y crear un modelo de responsabilidad mutua.

Desde un punto de vista institucional, AccountAbility (2008) alude al stakeholder engagement como el proceso por el cual una organización involucra a los grupos de interés más relevantes para alcanzar resultados aceptados. La 
La utilidad de los informes de sostenibilidad en entidades públicas y privadas en Colombia...

Organización de las Naciones Unidas para el Ambiente (PNUMA), la Asociación de Contadores Públicos Certificados - ACCA—, el Consejo de Estándares de Contabilidad para la Sostenibilidad de Estados Unidos - SASB - y el GRI han planteado que el stakeholder engagement es un requisito esencial para la generación de informes de sostenibilidad (O'Riordan y Fairbrass, 2014). También se plantea que es la base para la producción de información de sostenibilidad, porque a través de este se promueve la relevancia y materialidad de lo que debe ser informado (GRI, 2015b).

Dado que mediante el stakeholder engagement las organizaciones reciben retroalimentación por parte de sus stakeholders, sobre sus expectativas y los asuntos que consideran relevantes en materia de RSC y sostenibilidad, se ha considerado que este proceso es crucial, porque, con el mismo, se genera la impresión de que las organizaciones están siendo democráticas y socialmente responsables (Greenwood, 2007; Devin y Lane, 2014).

En la literatura se plantea que una de las principales funciones del stakeholder engagement, es establecer canales de comunicación entre los gestores y un rango amplio de stakeholders (Bebbington, Brown, Frame y Thomson, 2007; Bortree y Seltzer, 2009; Fasan y Mio, 2017). Esto implica que las organizaciones deben abandonar la idea unidireccional según la cual los grupos de interés son receptores pasivos de información. Por el contrario, los gestores deben comprender que la capacidad de opinión, crítica y juicio de los grupos de interés hacia las prácticas de información organizacional (Rinaldi, 2013), es vital para obtener una mejor comprensión de lo que afecta en mayor medida a los diversos stakeholders.

Recientemente, varios estudios señalan la importancia del stakeholder engagement para definir la materialidad de la información de sostenibilidad, puesto que representa un insumo fundamental para el cumplimiento de este principio contenido en los estándares GRI y para garantizar la utilidad de los reportes (Fasan y Mio, 2017; Ngu y Amran, 2018; Torelli, Balluchi y Furlotti, 2020; Almeida, Pinto y Fonseca, 2021). No obstante, una cuestión fundamental a considerar es que este proceso no inicia en el ámbito de las organizaciones que producen informes, sino que parte desde la estandarización que constituye el marco de referencia central para producir y divulgar dicha información. En este sentido, debe analizarse si los estándares, indicadores y directrices propuestas por organismos emisores como el GRI, se alinean con las expectativas y necesidades de los grupos de interés y les resultan de utilidad.

Este análisis es de importancia por cuanto la evolución del marco GRI ha implicado un cambio en su retórica institucional mediante la cual, de acuerdo con Grushina (2017), se ha fundado la noción de "diseño colaborativo" de los estándares, que promueve el imaginario según el cual los grupos de interés tienen una gran influencia en la determinación de lo que debe divulgarse por distintos 
tipos de organizaciones y en la manera como a nivel micro organizacional los stakeholders deben ser involucrados.

Manetti (2011) menciona que diversos estudios han cuestionado la sinceridad y el impacto de las prácticas de stakeholder engagement en los reportes de sostenibilidad, lo que implica que a pesar de que se ha introducido el discurso del diálogo con los stakeholders, existe poca evidencia que soporte que tal involucramiento ha tenido lugar en la realidad. Esto también podría ocurrir con la vinculación de los stakeholders en el proceso de emisión de guías y estándares. Por ello, Grushina (2017) identifica que desde el mismo proceso de estandarización pueden existir fuertes limitaciones para materializar la participación de stakeholders. Así, este trabajo retoma el análisis del stakeholder engagement, teniendo como foco central el proceso de estandarización del GRI.

\section{La metodología GRI para la definición de expectativas de los stakeholders}

Desde principios de 1990 , la contabilidad social y ambiental ha tomado un papel central en la generación de información para los stakeholders. Frente a ello, una de las organizaciones que ha ganado mayor reconocimiento en el desarrollo de un marco conceptual para la elaboración de información de sostenibilidad (Gómez y Quintanilla, 2012) y por emitir los estándares de mayor aceptación mundial (GRI, 2019a) es el Global Reporting Initiative. El enfoque multistakeholders del GRI ha tomado mayor protagonismo a partir de la guía G4, que ofreció los principios de contenido y calidad de la información en sostenibilidad, entre los que se encuentran la inclusión de los grupos de interés y la materialidad (GRI, 2014).

Dado que la relevancia, la materialidad y la inclusión, son principios clave para la generación de informes de sostenibilidad, entre 2012-2013 el GRI lanzó el proyecto denominado: "What do stakeholders want to know?" buscando documentar los asuntos más relevantes para la sostenibilidad, considerando cinco grupos de interés: asociaciones empresariales, representantes de los trabajadores; organizaciones de la sociedad civil, usuarios de la información y expertos, en un total de 52 industrias.

Dicho proyecto fue una referencia internacional sobre diversos temas, buscando aumentar la comparabilidad y la calidad de los reportes, e integrando el enfoque multistakeholder. No obstante, este proyecto no observó el debido proceso del GRI, por lo que no se realizó una consulta pública, una deliberación en grupos técnicos, ni se desarrolló un documento de bases para conclusiones. Por esto, es relevante considerar los resultados de este proyecto del GRI, que se relacionan directamente con los objetivos del presente trabajo, buscando sistematizar las expectativas y el involucramiento de los stakeholders en la información de sostenibilidad. 
La utilidad de los informes de sostenibilidad en entidades públicas y privadas en Colombia...

\section{II.1 El resumen de resultados del GRI y sus limitaciones frente al stakeholder engagement}

El 76\% de los temas considerados relevantes fue obtenido de la investigación interna realizada por el staff de GRI - en total se obtuvieron 2,812 temas-, siendo la mayoría de las organizaciones participantes, empresas e instituciones mediadoras y, en menor medida, organizaciones representantes de los trabajadores. Esto se sintetiza en la tabla 1.

Tabla 1. Número y Porcentaje de participantes por tipo de contribución.

\begin{tabular}{|c|c|c|c|c|c|c|}
\hline Tipo de contribución & 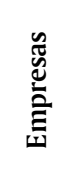 & $\begin{array}{c}\text { Organizaciones } \\
\text { de la sociedad } \\
\text { civil }\end{array}$ & $\begin{array}{l}\text { Mercados financieros } \\
\text { y usuarios de la } \\
\text { información }\end{array}$ & $\begin{array}{c}\text { Representantes } \\
\text { de los } \\
\text { trabajadores }\end{array}$ & 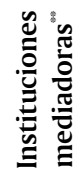 & $\begin{array}{l}\bar{\pi} \\
\overline{\tilde{J}} \\
\bar{\delta}\end{array}$ \\
\hline $\begin{array}{l}\text { Contribuciones externas } \\
\text { (directamente por } \\
\text { participantes) }\end{array}$ & 31 & 6 & 5 & 1 & 13 & 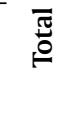 \\
\hline Investigación interna del GRI & 84 & 21 & 4 & 2 & 34 & \\
\hline Total & 115 & 27 & 9 & 3 & 47 & 201 \\
\hline Porcentajes & $57 \%$ & $13 \%$ & $4 \%$ & $1 \%$ & $23 \%$ & $100 \%$ \\
\hline
\end{tabular}

*GRI no distingue otro tipo de usuarios de la información, más allá de los financieros.

**:Las instituciones mediadoras se refieren, según el GRI, a organizaciones de expertos -consultores, instituciones académicas, gobiernos u organizaciones intergubernamentales.

Fuente: GRI (2013).

A pesar de que los usuarios de la información en mercados financieros solo representaron el $4 \%$ del total de participantes, la mayoría de los temas que resultaron relevantes fueron obtenidos de esta fuente (34\% del total de temas), seguidos por organizaciones de la sociedad civil ( $24,5 \%$ del total de temas) y por empresas ( $24 \%$ del total de temas). En este sentido, la preponderancia de la mirada financiera en aspectos de RSC contrasta con las declaraciones del GRI, sobre la necesidad de involucrar una perspectiva multistakeholder. A su vez, este dominio refleja que el GRI mantiene una postura alineada con las corrientes dominantes de la RSC enfocadas en la visión estratégica financiera y que implican la asimilación de la información en este ámbito, como un mecanismo útil para decisiones en los mercados (Gray y Collison, 2001).

Esto se corrobora con algunas publicaciones posteriores del GRI, que invocan el tema de la relevancia en la información de sostenibilidad. Por un lado, en 2015 el GRI publicó el primer documento de análisis de tendencias en el reporte de información de sostenibilidad. En este se destaca la generación de información en sostenibilidad con un enfoque en los intereses de los inversores. Así, el GRI se alinea con organizaciones como el Carbon Disclosure Project 
(CDP), el IIRC y el SASB, en la consideración de que "los inversionistas son los agentes más críticos, capaces de acelerar la transición hacia una economía sostenible"(GRI, 2015b, p. 9). Asimismo, en este documento se considera que los stakeholders son importantes, en tanto la tecnología les permite empoderarse y generar opiniones que pueden influir fuertemente en el mercado y en la regulación de diversos sectores (GRI, 2015b, 2015c, 2016b).

En esta misma línea, entre 2015 y 2016 el GRI aborda el concepto de materialidad, como un eje central para influir en las evaluaciones de los inversores sobre las compañías de su interés. Allí se define la materialidad como un proceso que identifica, prioriza, valida y comunica temas de sostenibilidad de acuerdo al principio de relevancia (GRI, 2015a, 2016a).

Bajo esta consideración, de acuerdo con Unerman y Zapettini (2014), los reportes de sostenibilidad fabrican una imagen de la realidad de la organización desde la visión de los stakeholders con mayor poder económico y capacidad para incidir (los inversores), por lo que, para comprender el contexto organizacional, es necesario observar lo que se divulga, pero también lo que se excluye de ser contado, a la vez que aumenta el cuestionamiento sobre quién decide qué es o no material.

Asimismo, a pesar de la importancia que representa la región latinoamericana en temas de sostenibilidad, por el alto índice de conflictos socioambientales que enfrenta (Rodríguez, 2016), ninguna organización proveniente de esta región fue considerada en el estudio, lo que evidencia una fuerte preponderancia de la tecnoregión del norte (Ariza, León y Gómez, 2008), agudizando la desigualdad y la deuda del crecimiento ${ }^{2}$ por parte del Norte con el Sur Global (Mosangini, 2012).

Los resultados del proyecto del GRI arrojan algunos puntos en común para varias industrias. Teniendo en cuenta que en Colombia las industrias consideradas líderes en sostenibilidad han sido la energética, la petrolera, la financiera, la de alimentos y la de comunicaciones (Gómez-Villegas y Quintanilla, 2012), hemos sintetizado en la tabla 2 los temas categorizados por el estudio de GRI (2013) como prioritarios para los grupos de interés. En esta investigación retomamos los temas que el proyecto del GRI (2013) ha identificado como relevantes, para preguntar a los stakeholders en Colombia por su percepción sobre los mismos. De igual forma, dado que desde 2015 los Objetivos del Desarrollo Sostenible (ODS) se presentan como una agenda prioritaria a nivel mundial, el GRI y Naciones Unidas han desarrollado acuerdos a través del SDG Compass, para que los estándares de sostenibilidad se encuentren alineados con estos y permitan que las organizaciones reflejen su contribución en este aspecto (GRI

2 Comprende el conjunto de responsabilidades no asumidas por el Norte Global, como la deuda ecológica, la deuda social, la deuda cultural, la deuda histórica y la deuda económica (Mosangini, 2012). 
La utilidad de los informes de sostenibilidad en entidades públicas y privadas en Colombia...

y UN Global Compact, 2017; GRI, UN Global Compact y WBCSD, 2015). Por ello, como se verá más adelante, nuestro estudio también indaga sobre la utilidad de este tipo de información.

Tabla 2. Temas relevantes por industrias-sectores.

\begin{tabular}{|c|c|c|c|c|c|c|}
\hline Tema /Sector & 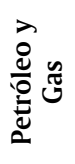 & 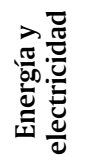 & 을 & 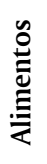 & $\stackrel{\cup}{F}$ & 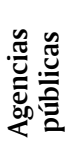 \\
\hline Accountability Político & $\mathrm{x}$ & $\mathrm{x}$ & $\mathrm{x}$ & $\mathrm{x}$ & $\mathrm{x}$ & \\
\hline Corrupción & $\mathrm{x}$ & $\mathrm{x}$ & $\mathrm{x}$ & $\mathrm{x}$ & & $\mathrm{x}$ \\
\hline Eficiencia energética & $\mathrm{x}$ & $\mathrm{x}$ & & & $\mathrm{x}$ & \\
\hline Emisiones (por GEI, diferentes al GEI) & $\mathrm{x}$ & $\mathrm{x}$ & $\mathrm{x}$ & $\mathrm{x}$ & $\mathrm{x}$ & \\
\hline Gobierno Corporativo & $\mathrm{x}$ & & $\mathrm{X}$ & $\mathrm{x}$ & $\mathrm{X}$ & $\mathrm{x}$ \\
\hline Salud Ocupacional y Seguridad para el empleado & $\mathrm{x}$ & $\mathrm{x}$ & $\mathrm{X}$ & $\mathrm{x}$ & $\mathrm{x}$ & \\
\hline Relaciones empleador-trabajador & $\mathrm{x}$ & & $\mathrm{x}$ & $\mathrm{x}$ & $\mathrm{x}$ & \\
\hline Prácticas de empleo & $\mathrm{x}$ & & $\mathrm{x}$ & $\mathrm{x}$ & & \\
\hline Uso y disposición de Materiales & & $\mathrm{x}$ & & $\mathrm{x}$ & $\mathrm{x}$ & \\
\hline Impactos económicos indirectos relacionados con servicios a la comunidad & $\mathrm{x}$ & $\mathrm{x}$ & & $\mathrm{x}$ & $\mathrm{x}$ & \\
\hline Derechos de los indíginas & $\mathrm{X}$ & & $\mathrm{x}$ & $\mathrm{x}$ & & \\
\hline Condiciones laborales & & & $\mathrm{x}$ & $\mathrm{x}$ & $\mathrm{x}$ & \\
\hline
\end{tabular}

Fuente: Elaboración propia, con base en GRI (2013).

Llaman la atención los resultados para agencias (entidades) públicas, pues solo se observan 2 temas considerados materiales, focalizados en cuestiones económicas y sociales — sin tener presencia directa la dimensión ambientalUna cuestión crítica de la clasificación realizada por el GRI, tiene que ver con que, para el caso de las agencias públicas, solamente se consideran los ministerios, las agencias federales, las entidades de gobierno regionales, las agencias de Estado, los ayuntamientos, las gobernaciones, y otro tipo de organizaciones que pertenecen a la estructura administrativa de los gobiernos. Con esto, las empresas de capital público o mixto quedan integradas, junto con aquellas de capital privado, lo que equipara en ambos casos sus fines y les prescribe los mismos objetivos e impactos en diversos stakeholders, aun cuando tales entidades también tienen como función el cumplimiento de objetivos misionales del Estado, mediante la prestación de servicios a los ciudadanos, lo que no implica, necesariamente, buscar la maximización del desempeño financiero (Cunningham, 2011; Rodríguez-Bolívar, Garde-Sánchez y López-Hernández, 2015).

\section{Metodología}

Para identificar las expectativas de los stakeholders en Colombia, diseñamos dos cuestionarios para aplicar vía web. Ambos fueron sometidos a pruebas 
piloto para evaluar su validez, comprensibilidad y coherencia. Uno de los cuestionarios fue dirigido a gestores y directivos, productores de políticas públicas, funcionarios públicos, asesores o personal encargado de la regulación, la producción y/o utilización de información cuantitativa y cualitativa de entidades del sector público; así como profesores y estudiantes de maestría y otros usuarios de la información contable. Otro cuestionario fue dirigido al grupo de interés del sector privado que incluyó a gestores y directivos, preparadores y auditores de información social y ambiental, inversores, reguladores de la información contable de este sector, profesores, estudiantes de maestría y otros usuarios de la información contable en Colombia. La figura 1 muestra el diseño de la encuesta.

Figura 1. Diseño de encuesta.

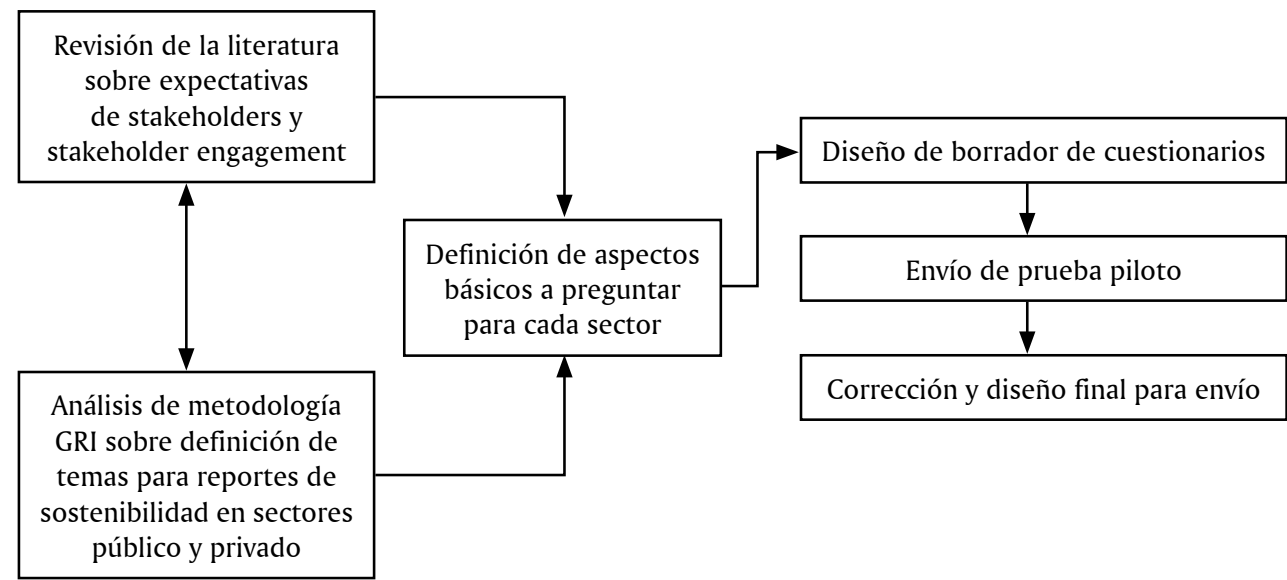

Fuente: Elaboración propia.

Debido al propósito de la encuesta y al enfoque cualitativo del estudio, la muestra de participantes fue deliberada. Este tipo de muestreo es plenamente aceptado en las investigaciones de este campo de estudio específico y tiene como criterios de selección la comprensión y pertinencia de los actores y no su representatividad estadística (Thorne, Mahoney y Manetti, 2014; Vaz Ogando, Ruiz Blanco, y Fernandez-Feijoo Souto, 2018; Xiao y Shailer, 2021). El cuestionario para el sector público fue enviado a 31 personas que cumplían los perfiles del grupo de interés, mientras que el cuestionario para el sector privado fue enviado a 69 personas que cumplían con los perfiles de dicho grupo, para un total de 100 personas. Ambos cuestionarios constaban de 12 preguntas. Las características de cada cuestionario pueden observarse en la tabla 3. Las encuestas fueron enviadas mediante correo electrónico con un protocolo que contenía una presentación del grupo de investigación, la explicación del propósito 
La utilidad de los informes de sostenibilidad en entidades públicas y privadas en Colombia...

de la encuesta, la aclaración de que el instrumento no requería identificación específica del encuestado y un vínculo al cuestionario online. El porcentaje de personas que respondieron la encuesta para el sector público fue del $74.19 \%$ y para el sector privado fue del $56.52 \%$. La tabla 4 relaciona los temas y las preguntas efectuadas. Las respuestas abiertas fueron tratadas mediante análisis de contenido (Bardin, 2002) usando Nvivo versión 11.

Tabla 3. Características de las encuestas por grupo de interés.

\begin{tabular}{lc}
\hline & Sector privado \\
\hline Herramienta online usada & $\begin{array}{c}\text { Survey Monkey versión } \\
\text { estándar mensual } \\
\text { Idioma }\end{array}$ \\
Periodo de captura de datos & Junio-Julio de 2019 \\
$\mathrm{~N}^{\circ}$ y tipo de preguntas & $12(1$ pregunta abierta para \\
& conocer el tipo de agente \\
encuestado -su relación \\
con el sector-; 4 preguntas \\
cerradas; 4 preguntas \\
cerradas con opción de \\
responder ¿por qué? y 3 \\
preguntas abiertas).
\end{tabular}

$\mathrm{N}^{\circ}$ de Respondientes
39
Sector público

Survey Monkey versión estándar mensual

Español

Junio-Julio de 2019

12 (1 pregunta abierta para conocer el tipo de agente encuestado -su relación con el sector-; 3 preguntas cerradas; 4 preguntas cerradas con opción a responder ipor qué?; 3 preguntas abiertas y 1 pregunta en la que debía añadirse si la afirmación aplicaba a agencias del gobierno, empresas con participación estatal-EPE- o ambas).

23

Fuente: Elaboración propia.

Tabla 4. Relación de Temas y Preguntas.

\begin{tabular}{|c|c|c|}
\hline Tema & Pregunta sector privado & Pregunta sector público \\
\hline \multirow{6}{*}{ 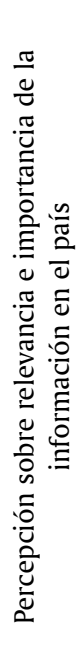 } & RQ 2 (ver más adelante) & RQ 2 (ver más adelante) \\
\hline & RQ 3 (ver más adelante) & RQ 3 (ver más adelante) \\
\hline & $\begin{array}{c}\text { RQ4 ¿Considera usted que los Objetivos de } \\
\text { Desarrollo Sostenible son un tema relevante } \\
\text { para las empresas? ¿Por qué? }\end{array}$ & $\begin{array}{c}\text { RQ } 4 \text { ¿Considera usted que los Objetivos de } \\
\text { Desarrollo Sostenible son un tema relevante } \\
\text { para las entidades públicas y las EPE? ¿Por qué? }\end{array}$ \\
\hline & RQ 9 (ver más adelante) & RQ 9 (ver más adelante) \\
\hline & $\begin{array}{l}\text { RQ } 10 \text { Por favor indique al menos cinco (5) } \\
\text { temas que no estén incluidos en la tabla } \\
\text { anterior y que usted esperaría que fuesen } \\
\text { informados en las Empresas privadas, } \\
\text { en materia de Responsablidad Social y } \\
\text { Medioambiental. }\end{array}$ & $\begin{array}{l}\text { RQ } 10 \text { Por favor indique al menos cinco (5) } \\
\text { temas que no estén incluidos en la tabla } \\
\text { anterior y que usted esperaría que fuesen } \\
\text { informados en las EPE, en materia de } \\
\text { Responsablidad Social y Medioambiental. }\end{array}$ \\
\hline & $\begin{array}{l}\text { RQ } 11 \text { Por favor indique al menos cinco (5) } \\
\text { temas que no estén incluidos en la tabla } \\
\text { anterior y que usted esperaría que fuesen } \\
\text { informados por las Agencias (entidades) } \\
\text { públicas - Gobierno, en materia de } \\
\text { Responsablidad Social y Medioambiental. }\end{array}$ & $\begin{array}{l}\text { RQ } 11 \text { Por favor indique al menos cinco (5) } \\
\text { temas que no estén incluidos en la tabla } \\
\text { anterior y que usted esperaría que fuesen } \\
\text { informados por las Agencias (entidades) } \\
\text { públicas - Gobierno, en materia de } \\
\text { Responsablidad Social y Medioambiental. }\end{array}$ \\
\hline
\end{tabular}




\begin{tabular}{|c|c|c|}
\hline Tema & Pregunta sector privado & Pregunta sector público \\
\hline \multirow{9}{*}{ 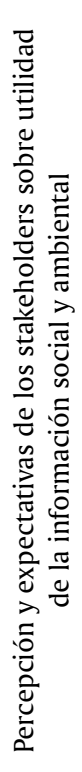 } & $\begin{array}{l}\text { RQ } 5 \text { ¿Considera usted que la información } \\
\text { social y medioambiental que producen las } \\
\text { empresas representa alguna posibilidad } \\
\text { de utilidad para las decisiones o para la } \\
\text { rendición de cuentas? }\end{array}$ & $\begin{array}{c}\text { RQ } 5 \text { ¿Considera usted que la información } \\
\text { social y medioambiental que deben producir } \\
\text { las entidades de gobierno es la misma que } \\
\text { deben producir las entidades que cuentan con } \\
\text { participación estatal? }\end{array}$ \\
\hline & RQ 6.1 (ver más adelante) & RQ 6.1 (ver más adelante) \\
\hline & RQ 6.2 (ver más adelante) & RQ 6.2 (ver más adelante) \\
\hline & RQ 6.3 (ver más adelante) & RQ 6.3 (ver más adelante) \\
\hline & RQ 6.4 (ver más adelante) & RQ 6.4 (ver más adelante) \\
\hline & RQ 6.5 (ver más adelante) & RQ 6.5 (ver más adelante) \\
\hline & RQ 6.6 (ver más adelante) & RQ 6.6 (ver más adelante) \\
\hline & RQ 7 (ver más adelante) & RQ 7 (ver más adelante) \\
\hline & $\begin{array}{l}\text { RQ } 12 \text { ¿Considera que los informes contables } \\
\text { y financieros deberían integrarse con la } \\
\text { información social y medioambiental, así } \\
\text { como otros tipos de información, para } \\
\text { conseguir una mirada holística de los procesos } \\
\text { y desempeño de las entidades o empresas? }\end{array}$ & $\begin{array}{l}\text { RQ } 12 \text { ¿Considera que los informes contables } \\
\text { y financieros deberían integrarse con la } \\
\text { información social y medioambiental, así como } \\
\text { otros tipos de información, para conseguir una } \\
\text { mirada holística de los procesos y desempeño } \\
\text { de las entidades o EPE? }\end{array}$ \\
\hline
\end{tabular}

\section{Resultados y Discusión}

\section{V.1 Perfil de los encuestados}

Para el sector privado recibimos 39 respuestas. La figura 2 resume los perfiles de quienes respondieron la encuesta para este grupo. La gran mayoría de quienes respondieron se desempeñan como preparadores de la información contable $(30.77 \% ; n=12)$, profesores universitarios $(23.08 \% ; n=9)$ y otros usuarios de la información contable $(17.94 \%$; $\mathrm{n}=7)$, estudiantes de máster en contabilidad y afines $(15.38 \%$; $n=6) \mathrm{y}$, en menor medida, auditores $(7.69 \%$; $n=3)$ y reguladores $(5.13 \% ; n=2)$.

Para el sector público recibimos 23 respuestas. La figura 3 muestra los perfiles de los respondientes. En su mayoría fueron los encargados de la producción de información en entidades públicas o empresas con participación estatal $(21.74 \% ; n=5)$, funcionarios públicos en otros cargos $(21.74 \% ; n=5)$ y estudiantes de máster (en contabilidad o afines —administración y tributación-) (17.39\%; $\mathrm{n}=4$ ). También participaron asesores contables y regulatorios de agencias gubernamentales $(13.04 \% ; n=3)$ y en menor medida profesores universitarios $(8.69 \% ; n=2)$, reguladores $(8.69 \% ; n=2)$, directivos $(4.35 \% ; n=1)$ y otros usuarios de la información en el sector $(4.35 \%$; $n=1)$. 
La utilidad de los informes de sostenibilidad en entidades públicas y privadas en Colombia...

Figura 2. Perfil de los respondientes del sector privado.

Fuente: Elaboración propia.

Figura 3. Perfil de los respondientes del sector público.

\section{Asesor \\ - Funcionario Público \\ - Profesor}

(1)

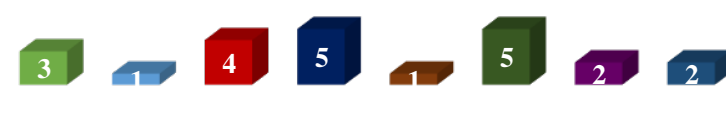

Directivo

- Otro usuario

- Regulador
Estudiante

- Producción información

Fuente: Elaboración propia.

\section{V.2 Percepción sobre la relevancia/ importancia de la información} para cada sector

La RQ2 buscó indagar sobre la percepción de los encuestados en términos de la importancia y relevancia de la información social y ambiental para cada sector. Los resultados para cada sector se sintetizan en la figura 4.

Figura 4. Percepción sector privado vs sector público frente a la afirmación "La información sobre asuntos sociales y ambientales de las empresas en el país es".

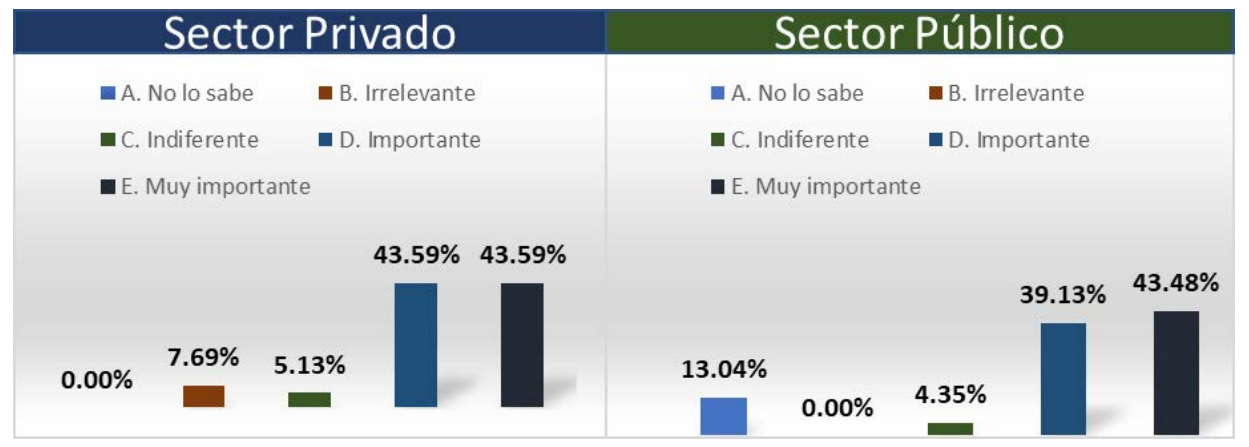

Fuente: Elaboración propia. 
En ambos sectores, la mayoría de encuestados considera que la información social y ambiental es importante (43,59\% para el sector privado y $39,13 \%$ para el sector público) o muy importante $(43,59 \%$ para el sector privado y $43,48 \%$ para el sector público). Una de las principales razones mencionadas para esta clasificación es la capacidad de informar sobre los impactos sociales y ambientales de la operación organizacional en cada sector. Otras razones expuestas para cada sector se ilustran en la tabla 5. Dentro de las razones que fueron más citadas para el sector privado se encuentra que esta información es un mecanismo de rendición de cuentas, y que aumenta la preocupación medioambiental en Colombia. En el sector público se esgrimieron razones, tales como que esta información se considera un mecanismo de rendición de cuentas, que es deber del Estado, que le permite ejercer control de sus instituciones y que es útil para la toma de decisiones de diversos actores entre los que se señalan empresas, ciudadanos y el gobierno.

Tabla 5. Razones sobre la importancia de la Información Social y Ambiental en los sectores privado y público.

\begin{tabular}{|c|c|c|c|}
\hline Sector privado & $\begin{array}{c}\text { Número de } \\
\text { referencias } \\
\text { asociadas }\end{array}$ & Sector Público & $\begin{array}{l}\text { Número de } \\
\text { referencias } \\
\text { asociadas } \\
\end{array}$ \\
\hline $\begin{array}{l}\text { Permite revelar los impactos } \\
\text { sociales y ambientales de la } \\
\text { operación }\end{array}$ & 15 & $\begin{array}{c}\text { Permite revelar los impactos sociales y } \\
\text { ambientales de la operación }\end{array}$ & 5 \\
\hline $\begin{array}{l}\text { Es un mecanismo de rendicición } \\
\text { de cuentas a grupos de interés }\end{array}$ & 9 & La rendición de cuentas es un deber del Estado & 4 \\
\hline $\begin{array}{l}\text { Exalta la preocupación } \\
\text { medioambiental en Colombia }\end{array}$ & 7 & $\begin{array}{c}\text { Permite el control estatal y social a las } \\
\text { instituciones de gobierno o con capital del } \\
\text { Estado }\end{array}$ & 4 \\
\hline $\begin{array}{l}\text { Es útil para la toma de decisiones } \\
\text { de grandes inversores }\end{array}$ & 1 & $\begin{array}{c}\text { Es útil para la toma de decisiones de empresas, } \\
\text { ciudadanos y del gobierno }\end{array}$ & 4 \\
\hline \multirow[t]{2}{*}{$\begin{array}{l}\text { Porque muestra el cumplimiento } \\
\text { en materia de ODS }\end{array}$} & 1 & $\begin{array}{l}\text { El cuidado social y ambiental son un deber y } \\
\text { una preocupación intrínseca del Estado }\end{array}$ & 4 \\
\hline & & $\begin{array}{l}\text { Es un insumo para el planteamiento de políticas } \\
\text { públicas }\end{array}$ & 2 \\
\hline
\end{tabular}

Fuente: Elaboración propia.

Por otro lado, en el sector privado el 7,69\% de los encuestados manifestó que esta información es irrelevante. Las razones expuestas son contradictorias. Algunos aducen que esta información no es obligatoria y, por tanto, no existe preocupación por parte de las empresas para informar sobre estos asuntos, mientras que otros aseguran que las empresas emiten información de este tipo solo cuando hay normas que las obligan, o para lavar su imagen.

Se destaca que para el sector privado hubo una referencia a que esta información es útil para mostrar el cumplimiento de las empresas en materia de 
La utilidad de los informes de sostenibilidad en entidades públicas y privadas en Colombia...

ODS. En relación con ello, la RQ4 preguntó a los encuestados de ambos sectores si consideraban que los ODS eran un tema relevante para las empresas y para las entidades públicas y empresas de participación estatal. En ambos casos, la mayoría de los encuestados contestaron afirmativamente, para el sector privado el $96 \%$ y para el sector público el $94.74 \%$. Gran parte de los encuestados del sector privado mencionó que los ODS fortalecen el compromiso de las organizaciones con la RSC y otros mencionaron que son un tema relevante porque buscan mitigar problemas mundiales como la pobreza y la desigualdad.

En el sector público, mayoritariamente los respondientes adujeron que los ODS representan el compromiso del Estado en la superación de problemas de la sociedad y el ambiente; otros mencionaron que éstos son relevantes porque coadyuvan en la definición de planes y programas de cara al futuro, permitiendo velar por el bien común y, en menor medida, los asociaron con preocupaciones ambientales que necesitan ser prioritarias para el Estado.

En cuanto a los estándares de referencia para la divulgación de información socioambiental, la RQ3 indagó a los participantes sobre las guías o estándares que están siendo usados por las empresas y por entidades de gobierno o por las Empresas con Participación Estatal, EPE, para la divulgación de este tipo de información. En su mayoría, los encuestados del sector privado reconocen los estándares del GRI como los de referencia para la divulgación de información social y ambiental $(38.3 \%$; $n=15)$ en empresas colombianas. Solo el $5.13 \%(n=2)$ señalaron que las empresas colombianas divulgan esta información con base en el modelo de Reporte Integrado. En contraste, en el sector público la mayoría de respondientes consideran que la divulgación de este tipo de información debería realizarse siguiendo el modelo de reporte integrado $(47.83 \%, \mathrm{n}=11)$, y el de los estándares del GRI $(30.43 \%, \mathrm{n}=7)$, lo que parece mostrar que ha existido cierta difusión del reporte integrado en el sector público en el país.

Por su parte, la RQ9 pidió a los participantes de ambos sectores que identificaran los temas que consideraban relevantes para los usuarios de la información social y ambiental de las empresas privadas, de las agencias o entidades públicas o del gobierno, y de las EPE. Los temas incluidos en la pregunta fueron tomados del conjunto de temas que resultaron del estudio del GRI antes mencionado. La figura 5 refleja las respuestas del sector privado.

En la figura 5 se evidencia que los encuestados del sector privado consideran que todos los temas incluidos son relevantes para los usuarios de la información, tanto en las empresas privadas como en las agencias públicas, con excepción de los derechos de los indígenas que solo representa un tema relevante para empresas privadas en el $48 \%$ de los respondientes. Este resultado contrasta con la gran cantidad de denuncias por la vulneración de los derechos a estas comunidades en Colombia, especialmente en sectores como la minería y el petróleo, fruto de conflictos ambientales en los que la empresa 
privada se ha visto como un actor fundamental, afectando su forma de vida, cultura, economía y cosmovisión (Rodríguez, 2016).

Figura 5. Temas relevantes para los usuarios de la información social y ambiental para empresas y agencias de gobierno - respuestas del grupo sector privado.

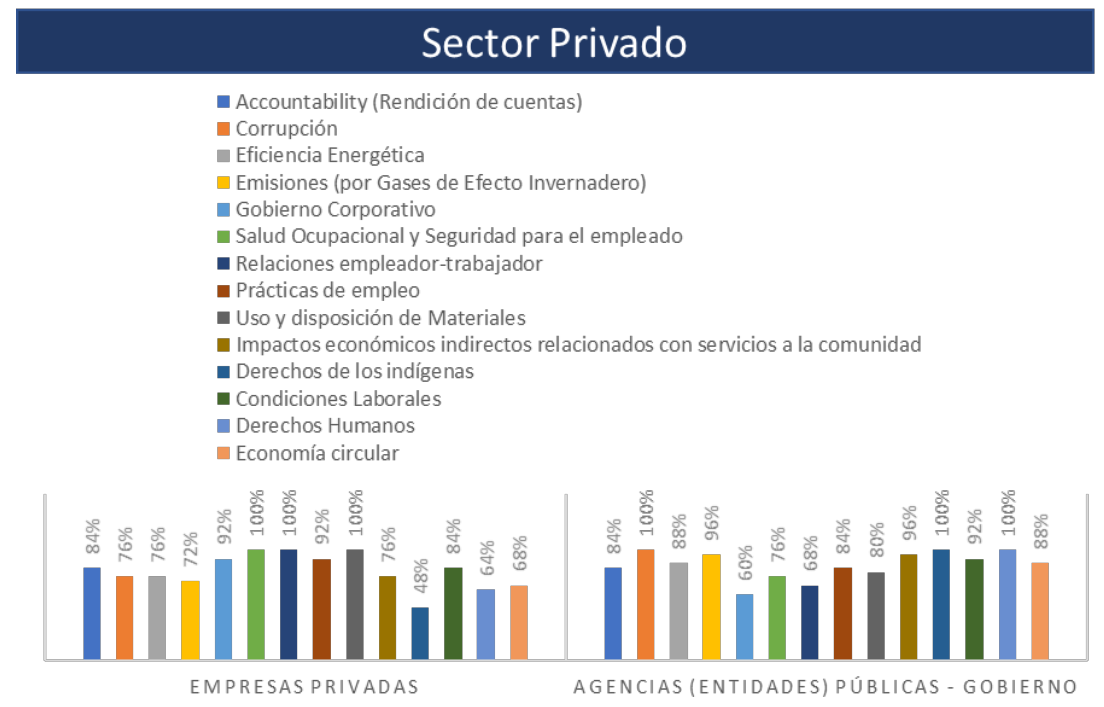

Fuente: Elaboración propia.

A manera de ejemplo, en el caso de El Cerrejón, los indígenas Wayuu se han visto afectados por el uso del agua por parte de la minera. De acuerdo con el Atlas de Justicia Medioambiental, la mina a cielo abierto allí situada consume más de 30 millones de litros de agua diarios, mientras que el acceso a agua por parte de los indígenas es altamente restringido (EJAtlas, 2019).

Rodríguez (2016) señala que los conflictos ambientales se caracterizan por baja participación y falta de información. Frente a ello, la información social y ambiental aumenta su relevancia en la medida en que contribuya a señalar las confrontaciones sociales, económicas y políticas de diversos actores, a la vez que permita a los usuarios de la información comprender los impactos directos e indirectos de la operación económica en la vida, la salud y el desarrollo de diversas comunidades.

En la misma línea de las respuestas obtenidas para el sector privado, los encuestados del sector público manifestaron que todos los temas expuestos son relevantes tanto para las EPE como para las agencias de gobierno. Aunque el tema de derechos de los indígenas fue señalado como relevante en el caso de las EPE, éste obtuvo el menor porcentaje de apoyo por parte de los respondientes $(63,16 \%)$, tal como se evidencia en la figura 6. 
La utilidad de los informes de sostenibilidad en entidades públicas y privadas en Colombia...

Figura 6. Temas relevantes para los usuarios de la información social y ambiental de empresas con participación estatal y agencias de gobierno - respuestas del grupo sector público.

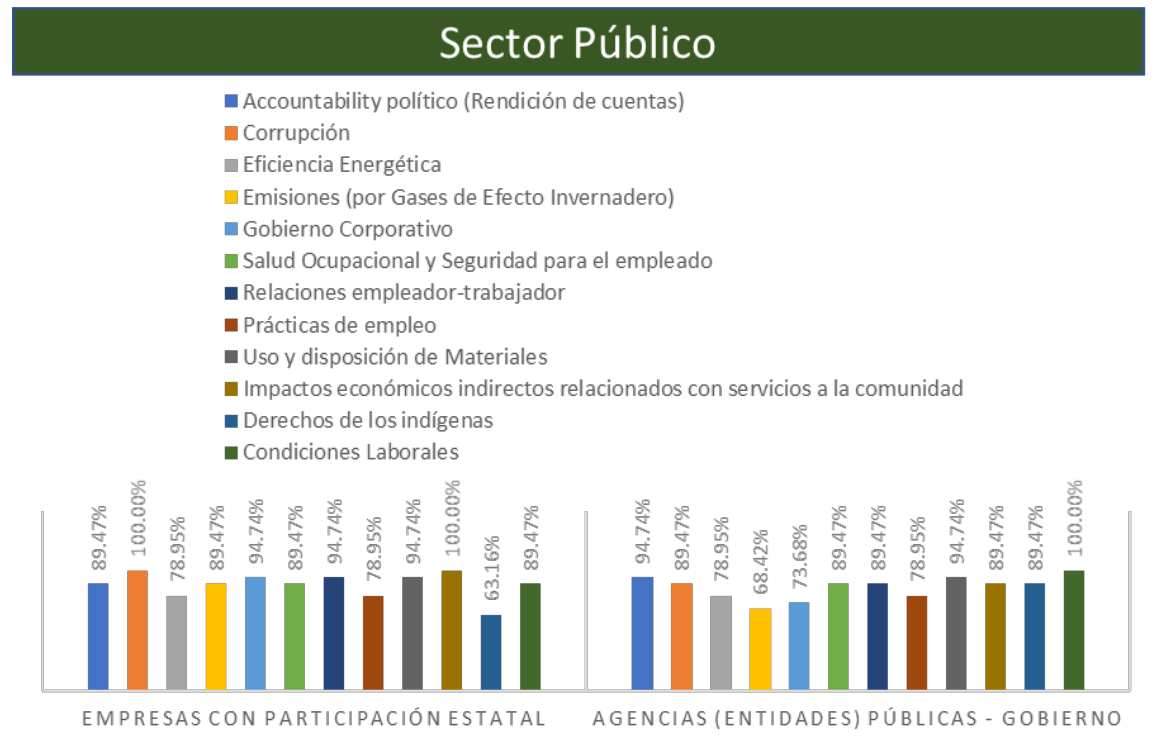

Fuente: Elaboración propia.

El caso de las EPE y los derechos de los indígenas también es objeto de tensiones en Colombia, puesto que la operación de diversas actividades económicas - sobre todo de EPE que se ocupan de la explotación de recursos naturales-, ocasiona efectos adversos en las condiciones de vida de estas comunidades. Uno de los ejemplos más representativos lo constituye el caso de la comunidad indígena de los U'was que, desde los años 2000, enfrentan una lucha contra Ecopetrol en Santander por recuperar tierras ancestrales y sagradas en las que la empresa tiene parte de sus operaciones (Rodríguez, 2016).

Teniendo en cuenta que la percepción de los grupos de interés es fundamental para establecer qué temas son relevantes y, por tanto, deben estar incluidos en los informes de sostenibilidad, la RQ10 pidió a los encuestados de ambos sectores, que señalaran al menos cinco (5) temas no mencionados en la encuesta y que deberían ser informados por parte de las empresas privadas y las EPE.

La tabla 6 agrupa los temas propuestos por los encuestados que no se encuentran reflejados en el estudio de GRI (2013) y que no aparecen expresamente en los estándares de dicha entidad. En este aspecto resaltan algunos cuyo alcance es más amplio que el de los estándares GRI y que, por tanto, reclamarían más información cualitativa. Al respecto, en la dimensión laboral se encontrarían temas como el clima organizacional, y la manera como se relacionan los objetivos de los empleados y de la compañía. En la dimensión social, específi- 
camente en cuanto a la comunidad, surgen temas como la manera en que las empresas han apoyado a las víctimas del conflicto y a los reinsertados. En la dimensión ambiental los encuestados señalan temas como la cultura medioambiental en la organización y los resultados de estudios ambientales. Finalmente, en la dimensión relacionada con el valor económico distribuido, uno de los temas más señalado es el de cumplimiento y transparencia en materia tributaria. $\mathrm{Al}$ respecto, GRI ha emitido el estándar 207 de impuestos, que debió iniciar su aplicación desde enero de 2021.

Tabla 6. Temas propuestos para el sector privado

\begin{tabular}{|c|c|c|c|}
\hline Temas propuestos para el sector privado & $\begin{array}{l}\text { Estándar } \\
\text { GRI }\end{array}$ & Tema Estándar & $\begin{array}{l}\text { Tratamiento } \\
\text { en el estándar }\end{array}$ \\
\hline$\%$ empleado en teletrabajo & & & No se trata \\
\hline Apoyo a la promoción laboral interna & & & No se trata \\
\hline Clima Organizacional & & & No se trata \\
\hline $\begin{array}{l}\text { Relación entre objetivos del trabajador y de la } \\
\text { compañía }\end{array}$ & & & No se trata \\
\hline Apoyo a víctimas del conflicto y a reinsertados & & & No se trata \\
\hline Cultura Medioambiental en la Organización & & & No se trata \\
\hline Resultado de estudios de impacto ambienatl & & & No se trata \\
\hline $\begin{array}{l}\text { Cumplimiento tributario, transparencia en materia } \\
\text { tributaria }\end{array}$ & & & No se trata \\
\hline Innovación en procesos y producción limpia & 102 & Divulgación General & Indirectamente \\
\hline $\begin{array}{l}\text { Asignación salarial/honorarios a personal clave en } \\
\text { materia de control como Revisoría Fiscal }\end{array}$ & 102 & Divulgación General & Indirectamente \\
\hline Estategias de mejoramiento Social y Ambiental & 102 & Divulgación General & Indirectamente \\
\hline Nivel de cumplimiento de ODS & 102 & Divulgación General & Indirectamente \\
\hline $\begin{array}{l}\text { Nivel de participación de empleados y stakeholders } \\
\text { en decisiones }\end{array}$ & $102 / 413$ & $\begin{array}{l}\text { Divulgación General/ } \\
\text { Comunidades Locales }\end{array}$ & Indirectamente \\
\hline Índice de transparencia & 205 & Prácticas anticorrupción & Indirectamente \\
\hline \% arborización & 304 & Biodiversidad & Indirectamente \\
\hline Efectos Ambientales Positivos & 304 & Biodiversidad & Indirectamente \\
\hline Respeto por diversidad de género & 405 & $\begin{array}{l}\text { Diversidad e igualdad } \\
\text { de oportunidades }\end{array}$ & Indirectamente \\
\hline Resultados de consultas prevías & 413 & Comunidades Locales & Indirectamente \\
\hline $\begin{array}{l}\text { Índice de calidad de vida - bienestar las } \\
\text { comunidades antes y durante operación }\end{array}$ & 413 & Comunidades Locales & Indirectamente \\
\hline
\end{tabular}

Fuente: Elaboración propia.

Otros temas sugeridos por los encuestados del sector privado se abordan de forma indirecta en los estándares de GRI. Teniendo en cuenta la particularidad del contexto colombiano, sería necesario incorporarlos para aumentar la capa- 
La utilidad de los informes de sostenibilidad en entidades públicas y privadas en Colombia...

cidad informativa de los reportes de sostenibilidad. Por ejemplo, en el ámbito de los contenidos generales, los encuestados han señalado la importancia de informar sobre aspectos como la asignación salarial o de honorarios a personal clave para el control de la organización, más allá de los miembros del Gobierno Corporativo. En este caso en particular se evidencia la necesidad de informar sobre los honorarios al Revisor Fiscal o al Auditor Externo.

Adicionalmente, aunque varias empresas líderes en información social y ambiental en Colombia han manifestado su deseo de contribuir al logro de los ODS, los encuestados señalan que es necesario expresar específicamente cuál ha sido el aporte directo de las organizaciones y cuál ha sido su nivel de cumplimiento. Un análisis de los informes integrados de empresas líderes en sostenibilidad en Colombia, como Argos y Nutresa, muestra que en 2018 y 2019 se ha divulgado de manera específica su contribución con algunos de los ODS (Argos S.A., 2018, 2019; Grupo Nutresa, 2018, 2019).

Los encuestados también señalaron que las empresas privadas deberían informar cuál ha sido el nivel de participación de los grupos de interés en las decisiones organizacionales. Esto se extiende más allá de lo dispuesto en los estándares y principios de GRI, que permanecen muy generales en este ámbito. En el estándar 102 de GRI se exige la divulgación del enfoque para la participación de los grupos de interés, pero, restringido a que la organización divulgue solo el medio por el cual se comunica con los diversos grupos mediante canales como encuestas, grupos focales, paneles o comunicados de la organización (Global Reporting Initiative, 2016).

Una revisión a los informes de sostenibilidad e integrados de las empresas líderes, tanto del sector privado como con participación estatal en Colombia durante los años 2016-2018 —Cementos Argos, Nutresa, EPM, Ecopetrol y Bancolombia - evidenció que, en realidad, lo que se divulga no es la manera en la que los grupos de interés participan en las decisiones estratégicas, sino la forma en que se les consulta - pasivamente- a algunos stakeholders sobre algunos temas específicos. Incluso, varios de estos informes se limitan a mencionar que cumplen con la metodología GRI de relacionamiento, sin explicar adecuadamente la manera como se realiza y sin aportar evidencia.

Uno de los temas cruciales en Colombia es la terminación del conflicto armado y la paz. Frente a ello, desde el año 2016 se ha resaltado el papel fundamental que tiene la empresa privada en el posconflicto (Cabrera-Narváez y Quinche-Martín, 2021). Por tal motivo, varios encuestados señalaron que los informes de sostenibilidad de las empresas deberían informar sobre cómo han apoyado a las víctimas del conflicto y a los reinsertados de los grupos armados. Este aspecto enfatiza en la necesidad de que los informes de sostenibilidad respondan directamente a ciertas especificidades del contexto. 
En cuanto a los encuestados del sector público, se evidenció un número menor de temas que no se encontraron en los estándares ni en el estudio del GRI y que son propuestos para agencias públicas. La RQ11 pidió a los encuestados de las empresas privadas que señalaran cinco temas que deberían ser incluidos en la información social y ambiental de las agencias estatales. Las respuestas de la RQ 10 en el grupo de encuestados del sector público y la RQ11 por el grupo de encuestados del sector privado se resume en la tabla 7 .

Dentro de los temas propuestos por los encuestados del sector público se encuentra la inclusión de cuentas ambientales. Varias respuestas señalaron la necesidad de que la información social y ambiental de las agencias de gobierno incluya información contable que permita ejercer un control de la gestión ambiental de cada organización, debido a que, actualmente en Colombia, las cuentas de recursos naturales y de medio ambiente no son ampliamente divulgadas por estas organizaciones.

Otro tema que fue señalado por los encuestados y que amerita un análisis de la interrelación entre los dos sectores, es el de los impactos o efectos que tienen los incentivos fiscales (beneficios tributarios en forma de deducciones y descuentos tributarios que disminuyen la obligación tributaria) por el desarrollo de programas sociales y ambientales. En los últimos años en Colombia se han promovido incentivos tributarios orientados a "premiar" la sostenibilidad y la RSC. Frente a ello, cada vez más empresas privadas han internalizado a la RSC dentro de sus modelos de planeación tributaria (Arias, Espitia y Ruíz, 2016; Cruz, Vélez y Romero, 2020) lo que redunda en una disminución en el recaudo de impuestos que posteriormente tiene impactos en el desarrollo de políticas sociales y ambientales por parte del Estado.

Tabla 7. Temas propuestos para el sector público

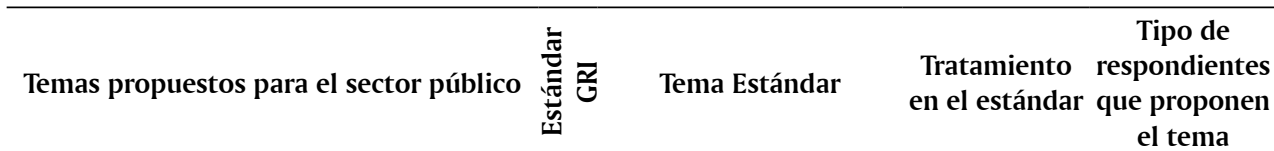

Cuentas ambientales (activos, pasivos, costos ambientales)

Impactos en programas públicos sociales y

No se trata Sector Público

ambientales por deducciones y descuentos

No se trata Sector Público

de impuestos otorgados al sector privado

para materialización de acuerdo de paz,

ambiente, etc.

Regalías obtenidas

No se trata Sector Público

Planes de recuperación ambiental en el

No se trata Sector Público

entorno y progarmas de investigación para

la mitigación de impactos 
La utilidad de los informes de sostenibilidad en entidades públicas y privadas en Colombia...

\begin{tabular}{|c|c|c|c|c|}
\hline Temas propuestos para el sector público & 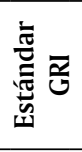 & Tema Estándar & $\begin{array}{c}\text { Tratamiento } \\
\text { en el estándar }\end{array}$ & $\begin{array}{l}\text { Tipo de } \\
\text { respondientes } \\
\text { que proponen } \\
\text { el tema } \\
\end{array}$ \\
\hline $\begin{array}{l}\text { \% de inversión de impuestos } \\
\text { mediambientales }\end{array}$ & & & No se trata & Sector Público \\
\hline $\begin{array}{l}\text { Número y cargos de funcionarios juzgados } \\
\text { por delitos y escándalos de corrupción en } \\
\text { la entidad }\end{array}$ & & & No se trata & Sector Público \\
\hline $\begin{array}{l}\text { Índice de bienestar de funcionarios y } \\
\text { servidores públicos }\end{array}$ & & & No se trata & Sector Público \\
\hline $\begin{array}{l}\text { Resultados de consultas previas con } \\
\text { comunidades }\end{array}$ & & & No se trata & Sector Público \\
\hline $\begin{array}{l}\text { Afiliación política de directivos de } \\
\text { entidades de gobierno }\end{array}$ & & & No se trata & Sector Público \\
\hline $\begin{array}{l}\text { Iniciativas de protección al Patrimonio } \\
\text { Cultural }\end{array}$ & & & No se trata & Sector Público \\
\hline Lobbying por parte de empresas privadas & & & No se trata & Sector Público \\
\hline $\begin{array}{l}\text { Tipo de vinculación del personal } \\
\text { (Funcionarios de planta, contratistas) }\end{array}$ & & & No se trata & Sector Público \\
\hline Nivel de cumplimiento de los ODS & 102 & Contenidos Generales & Indirectamente & Ambos \\
\hline $\begin{array}{l}\text { Opinión de grupos de interés sobre la } \\
\text { actuación en materia de sostenibilidad de } \\
\text { la organización }\end{array}$ & 102 & Contenidos Generales & Indirectamente & Sector Público \\
\hline $\begin{array}{l}\text { Capacitación en la entidad sobre temas } \\
\text { sociales y ambientales }\end{array}$ & 404 & Formación y Educación & Indirectamente & Sector Público \\
\hline $\begin{array}{l}\text { Políticas para apoyo a la población } \\
\text { vulnerable, víctimas de conflicto y } \\
\text { reinsertados }\end{array}$ & 413 & Comunidades Locales & Indirectamente & Sector Público \\
\hline
\end{tabular}

Fuente: Elaboración propia.

Uno de los temas que llama la atención en cuanto a la transparencia de la gestión pública propuesto por los encuestados, es el número y nivel de funcionarios que han sido investigados y juzgados por delitos. Aunque el estándar 205 del GRI aborda las estrategias anticorrupción, no contempla la divulgación de este tema, el cual es necesario en nuestro contexto, dada la corrupción percibida en el sector público - en el índice de transparencia internacional Colombia tiene 37/100 puntos ${ }^{3}$ - (Transparencia Internacional, 2020).

Otros temas propuestos, tales como el nivel de cumplimiento de los ODS, se encuentran contemplados de manera indirecta en los estándares y, de acuerdo con la opinión de los encuestados, necesitan de una divulgación mucho más amplia. Algo similar sucede con el tema de política de apoyo a población vulne-

3 En una escala de cero (0) sobre cien (100), en la que cero es altamente corrupto y cien significa sin corrupción. 
rable, víctimas del conflicto y reinsertados, pues con la firma de los acuerdos de paz se espera que las entidades de gobierno trabajen en la materialización de estos y contribuyan a una paz estable y duradera.

Los encuestados del sector privado consideran necesario que se divulgue la manera en la que las agencias de gobierno han aplicado los recursos recibidos como aportes para la materialización de los acuerdos de paz, o para la promoción de programas de cuidado ambiental, entre otros. Adicionalmente, teniendo en cuenta que en Colombia hay un nivel alto de explotación de recursos naturales que deben traducirse en pagos por regalías, los encuestados señalaron que debe incluirse información sobre este aspecto en los informes de sostenibilidad de las agencias de gobierno. Además, los encuestados del sector privado proponen que las entidades del Estado divulguen las iniciativas de protección al patrimonio cultural, y, para el caso de entidades encargadas de emisión de leyes — como el Congreso de la República-, el lobbying ejercido por empresas privadas.

El tema de los impuestos - recaudados y dejados de recaudar por los beneficios otorgados-, es un tema álgido para ambos sectores. En este caso, los encuestados señalaron la necesidad de que las agencias de gobierno informen cómo han invertido los ingresos por impuestos medioambientales.

\section{V.3 Percepción y expectativas sobre la utilidad de la información social y ambiental}

Indagamos a los encuestados por la utilidad de la información social y ambiental, por sus expectativas frente al papel de esta información en las organizaciones y por la necesidad de su regulación. En este sentido, la RQ5 preguntó a los participantes del sector privado si esta información sería de utilidad para la toma de decisiones o para la rendición de cuentas. Ninguno de los encuestados expresó una respuesta negativa. Sin embargo, de acuerdo con la figura 7 , los respondientes consideran que este tipo de información es más útil para la toma de decisiones $(n=17)$ que para la rendición de cuentas $(n=8)$. Este resultado contrasta con la literatura que resalta el potencial de esta información para la accountability (Cooper y Owen, 2007; Gray, 2007, 2010), y con lo expresado por el GRI al identificar en los suplementos sectoriales que la rendición de cuentas es una de las razones para la producción de reportes de sostenibilidad (GRI, 2013; Greco, Sciulli y D’Onza, 2015). Pero también evidencia el posicionamiento de la retórica de la utilidad de la información para la toma de decisiones (Ball, 2013). 
La utilidad de los informes de sostenibilidad en entidades públicas y privadas en Colombia...

Figura 7. ¿Considera usted que la información social y medioambiental que producen las empresas representa alguna posibilidad de utilidad para las decisiones o para la rendición de cuentas?

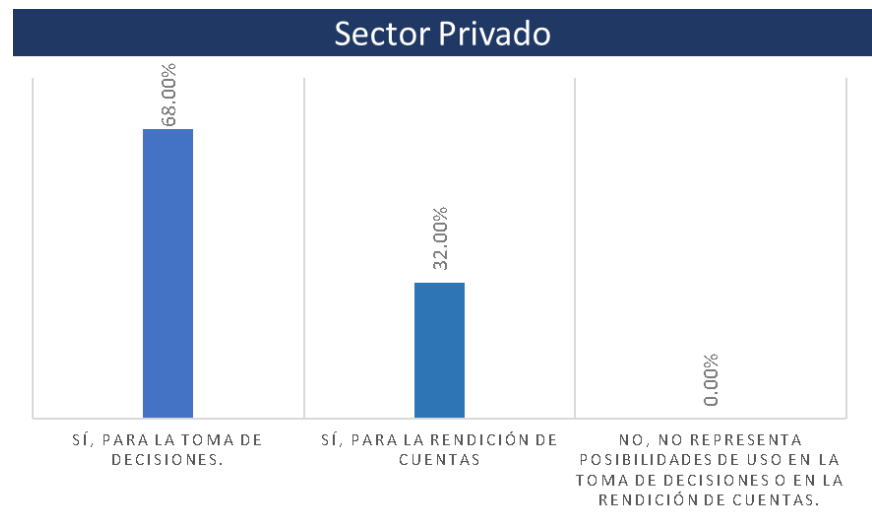

Fuente: Elaboración propia.

En cuanto al sector público, la RQ 5 preguntó si la información social y medioambiental que deben producir las entidades de gobierno es la misma que deben producir las empresas con participación estatal. El 73.68\% de los respondientes contestaron afirmativamente, tal como se observa en la figura 8. Siguiendo a Dumay, Guthrie y Farneti (2010) esta percepción podría promover la reproducción de lógicas propias de empresas del sector privado, en el sector público a través del uso de estándares diseñados desde una lógica instrumental de la RSC y la sostenibilidad.

Figura 8. ¿Considera usted que la información social y medioambiental que deben producir las entidades de gobierno es la misma que deben producir las entidades que cuentan con participación estatal?

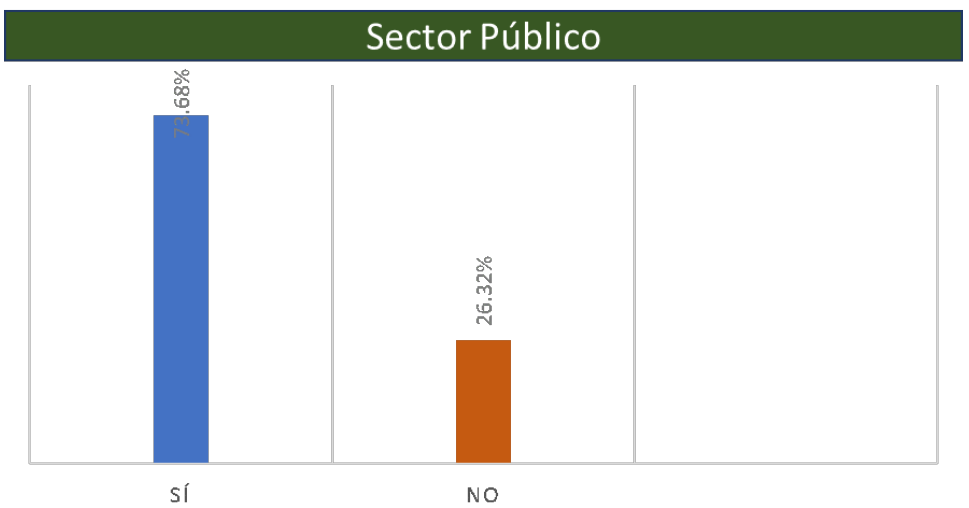

Fuente: Elaboración propia. 
Por otro lado, la pregunta 6 pedía que los encuestados manifestaran su posición afirmativa o negativa frente a seis afirmaciones relacionadas con la utilidad percibida, así como sus expectativas sobre la información social y ambiental; las afirmaciones se sintetizan en la tabla 8.

Tabla 8. Afirmaciones constituyentes de la RQ6

\begin{tabular}{|c|c|c|c|}
\hline \multicolumn{2}{|r|}{ Sector Privado } & \multicolumn{2}{|r|}{ Sector Público } \\
\hline $\mathbf{N}^{\circ}$ & Afirmación & $\mathbf{N}^{\circ}$ & Afirmación \\
\hline 6.1 & $\begin{array}{l}\text { Los asuntos sociales y medioambientales } \\
\text { deben ser un objetivo prioritario para las } \\
\text { empresas. }\end{array}$ & 6.1 & $\begin{array}{c}\text { Los asuntos sociales y medioambientales } \\
\text { deben ser un objetivo prioritario para los } \\
\text { gobiernos o las empresas con participación } \\
\text { estatal (EPE). }\end{array}$ \\
\hline 6.2 & $\begin{array}{l}\text { La información social y medioambiental } \\
\text { afecta directa o indirectamente a la } \\
\text { generación de resultados empresariales. }\end{array}$ & 6.2 & $\begin{array}{l}\text { La información social y medioambiental afecta } \\
\text { directa o indirectamente a la generación de } \\
\text { resultados en las entidades o EPE. }\end{array}$ \\
\hline 6.3 & $\begin{array}{c}\text { Revelar información social y } \\
\text { medioambiental permite identificar los } \\
\text { distintos grupos de interés y servir mejor a } \\
\text { sus necesidades. }\end{array}$ & 6.3 & $\begin{array}{l}\text { Revelar información social y medioambiental } \\
\text { permite identificar los distintos grupos de } \\
\text { interés y servir mejor a sus necesidades. }\end{array}$ \\
\hline 6.4 & $\begin{array}{l}\text { Revelar información social no es un tema } \\
\text { relevante cuando funciona adecuadamente } \\
\text { la junta directiva dentro de la compañía. }\end{array}$ & 6.4 & $\begin{array}{l}\text { Revelar información social y medioambiental } \\
\text { no es un tema relevante cuando funciona } \\
\text { adecuadamente la junta directiva dentro de } \\
\text { empresas con participación estatal. }\end{array}$ \\
\hline 6.5 & $\begin{array}{l}\text { Conocer información social y } \\
\text { medioambiental de una empresa permite } \\
\text { aproximarse mejor al valor de una empresa } \\
\text { y a su imagen fiel, frente a las valoraciones } \\
\text { que atienden solamente a la información } \\
\text { contable-financiera. }\end{array}$ & 6.5 & $\begin{array}{l}\text { Conocer información social y medioambiental } \\
\text { de una entidad o una EPE permite } \\
\text { aproximarse mejor y a su imagen fiel, antes } \\
\text { que atendiendo solamente a la información } \\
\text { contable-financiera. }\end{array}$ \\
\hline 6.6 & $\begin{array}{l}\text { Debería existir una regulación clara y } \\
\text { precisa, de carácter obligatorio, respecto a } \\
\text { la información social y medioambiental de } \\
\text { las empresas }\end{array}$ & 6.6 & $\begin{array}{c}\text { Debería existir una regulación clara y } \\
\text { precisa, de carácter obligatorio, respecto a } \\
\text { la información social y medioambiental de } \\
\text { las entidades de gobierno y las empresas con } \\
\text { participación Estatal (EPE) }\end{array}$ \\
\hline
\end{tabular}

Fuente: Elaboración propia.

La figura 9 refleja las respuestas de ambos sectores. La posición fijada por los encuestados respecto a la afirmación 6.1, soporta los resultados del grupo de preguntas sobre la importancia de la información social y ambiental, y contradice el sentido de las prácticas actuales de producción de información predominantemente financiera. En la misma línea, los resultados en cuanto a la afirmación 6.2 reflejan que la totalidad de encuestados del sector privado $(n=25)$ y la mayoría del sector público $(n=16)$ consideran que este tipo de información tiene un impacto directo en los resultados financieros de empresas privadas, empresas con participación estatal y agencias del gobierno, lo que 
La utilidad de los informes de sostenibilidad en entidades públicas y privadas en Colombia...

explicaría desde un punto de vista funcionalista, la necesidad de priorizar la divulgación de este tipo de información para favorecer la toma de decisiones, tal como se observó en la RQ5.

Figura 9. Repuestas a la $R Q 6$ sector privado vs sector público

\section{Sector Privado}

\section{Sector Público}

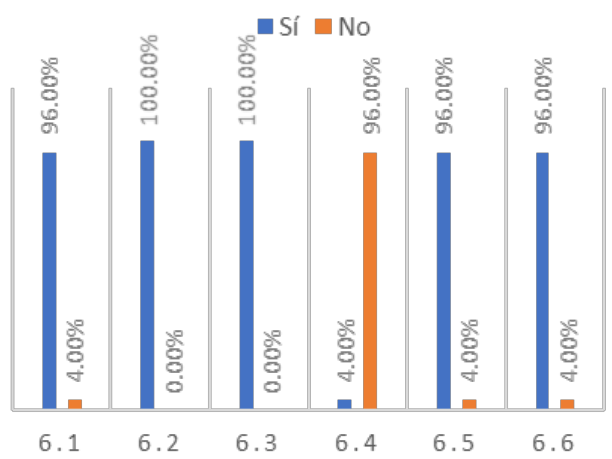

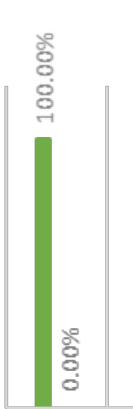

6.1
Sí $\square$ No

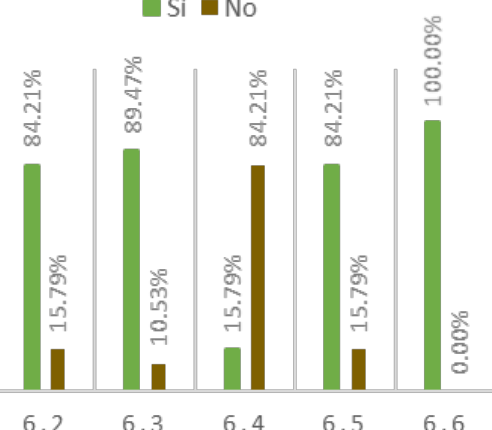

Fuente: Elaboración propia.

En cuanto a las afirmaciones 6.3 y 6.5, los respondientes de ambos sectores fueron enfáticos en manifestar que la información social y ambiental es útil para identificar a los grupos de interés y atender a sus necesidades, y permite una mejor aproximación a la imagen fiel de las empresas, entidades y EPE, aunque en el caso de los participantes del sector privado tal postura fue mucho más contundente que la del sector público (en este caso, el 10.53\% de los respondientes señalaron que la información social y medioambiental no es útil para identificar grupos de interés y responder a sus expectativas, y el $15.79 \%$ señaló que esta información no permite aproximarse mejor a la imagen fiel de las entidades de gobierno o de las EPE).

Estas respuestas son coherentes con la postura negativa manifestada por los respondientes frente a la afirmación 6.4. El 96\% de encuestados del sector privado y el $84.21 \%$ del sector público opinan que la información social y ambiental no pierde relevancia, aun si la junta directiva de las compañías o EPE opera adecuadamente.

La importancia que los encuestados otorgan a la información social y ambiental, tanto para usuarios financieros como no financieros, podría ser la base que soporte la expectativa de que esta sea regulada de manera obligatoria, como se aprecia en las posturas respecto de la afirmación 6.6. Esto contradice la corriente de voluntarismo tras la aplicación de estándares como los del GRI, que permiten la discrecionalidad en cuanto a cantidad y calidad de la 
información a divulgar. La discusión sobre la regulación de la información de sostenibilidad ha ganado particular importancia en los últimos años y nuestra investigación se alinea, de forma anticipada, con la consulta pública que realizó desde septiembre de 2020 la fundación IFRS sobre este aspecto (IFRSF, 2020).

Finalmente, en cuanto al tipo de usuarios que podrían ser los más interesados en la información social y ambiental producida por empresas, entidades públicas o EPE, la tabla 9 refleja que los encuestados del sector privado consideran que los bancos y otros financiadores serían el grupo más interesado, seguido de los trabajadores y sindicatos - lo que podría explicar la gran cantidad de temas expresados en la RQ 10 en cuanto al aspecto laboral-, y de los auditores financieros. Llama la atención a este respecto que los reguladores, las ONG y los inversores obtuvieron el menor puntaje, siendo catalogados como los menos interesados en este tipo de información en el sector privado, lo que se corresponde parcialmente con lo expresado por los encuestados del sector público que consideraron a los Inversores y las ONG como los usuarios menos interesados. En cuanto a las ONG, estos resultados contradicen estudios que las ven como actores sumamente relevantes e interesados en cuanto a la información divulgada por empresas privadas y entidades de gobierno (Amran y Ooi, 2014; Ver, por ejemplo, O’Dwyer, Unerman y Hession, 2005; Tilt, 2007). Estos resultados también van en línea con las respuestas de la RQ5 y dan cuenta de que la información social y ambiental está siendo insertada en las lógicas de la contabilidad financiera tradicional — siendo vista como útil para toma de decisiones de proveedores de capital, más que como una herramienta de rendición de cuentas- (IFRS, 2020).

Los encuestados del sector público señalaron que los gestores públicos, los funcionarios públicos y los organismos de control fiscal serían los grupos más interesados en la información social y ambiental divulgada por entidades de gobierno y EPE. En este sentido, llama la atención que los participantes no consideraran a los ciudadanos como principales interesados. Al respecto, Dumay, Guthrie y Farnetti (2010) han señalado que la emisión de información social y ambiental del sector público ha usado los parámetros de empresas privadas, principalmente a través de los estándares GRI, sin contar con participación alguna del público, lo que ha puesto en entredicho su relevancia para los ciudadanos. En este sentido, sugieren que, particularmente en el sector público, deberían usarse narrativas que permitan acercar la información social y ambiental al colectivo social, más que métricas abstractas, y estándares que son usados como discursos "managerialistas" a usar por los gestores públicos. 
La utilidad de los informes de sostenibilidad en entidades públicas y privadas en Colombia...

Tabla 9. Percepción sobre principales usuarios interesados en la información social y ambiental sector privado vs sector público

\begin{tabular}{|c|c|c|c|}
\hline Grupos de interés sector privado & Score & Grupos de interés sector público & Sector \\
\hline Bancos y otros financiadores & 6.92 & Gestores Públicos & 6.37 \\
\hline Trabajadores, sindicatos & 6.64 & Funcionarios Públicos & 6.05 \\
\hline Auditores financieros & 6.52 & Organismos de control fiscal-Contralorías & 5.79 \\
\hline Clientes & 6.04 & $\begin{array}{l}\text { Cuerpos políticos legislativos } \\
\text { (Congreso, Asambleas, Consejos) }\end{array}$ & 5.53 \\
\hline Ciudadanos & 5.76 & $\begin{array}{l}\text { Organismos intergubernamentales } \\
\text { (P.E ONU, OCDE, OEA, etc.) }\end{array}$ & 5.47 \\
\hline Gerentes & 5.32 & $\begin{array}{l}\text { Agencias de políticas públicas en el país } \\
\text { (Ministerios, DNP, otros). }\end{array}$ & 5.42 \\
\hline $\begin{array}{c}\text { Organismos intergubernamentales y otras } \\
\text { iniciativas (P.E., ONU, UNCTAD, GLOBAL } \\
\text { COMPACT). }\end{array}$ & 4.64 & Ciudadanos & 5.32 \\
\hline $\begin{array}{c}\text { Reguladores y otros organismos (CTCP, CGN, } \\
\text { otros). }\end{array}$ & 4.56 & $\begin{array}{c}\text { Organismos Internacionales de Crédito (P.E., } \\
\text { BM, FMI, BID, etc.) }\end{array}$ & 5.26 \\
\hline $\begin{array}{c}\text { Organizaciones no gubernamentales } \\
\text { promotoras de protección social y } \\
\text { ambiental. }\end{array}$ & 4.36 & Inversores & 5.16 \\
\hline Inversores & 4.24 & $\begin{array}{l}\text { Organizaciones no gubernamentales } \\
\text { promotoras de protección social y ambiental. }\end{array}$ & 4.63 \\
\hline
\end{tabular}

Fuente: Elaboración propia.

Finalmente, frente a la necesidad de constituir una mirada holística de las organizaciones, y en relación con la RQ 12, la mayoría de encuestados de los dos grupos consideran que es necesario realizar la integración de la información contable financiera con la información contable social y ambiental (para el sector privado 95.83\%; $\mathrm{n}=23$, mientras que para el sector público el $84.21 \%$; $\mathrm{n}=16$ apoyan esta consideración). En esta línea, Humphrey, O'Dwyer y Unerman (2017) han planteado que el International Integrated Reporting Council (IIRC) ha intentado institucionalizar el reporte integrado como una práctica relevante para la reconfiguración del reporte contable corporativo. No obstante, Deegan (2020) y Flower (2020) son críticos, al manifestar que el reporte integrado no logra mejorar la rendición de cuentas para un grupo amplio de stakeholders y mantiene los mismos problemas de otros modelos, ya que se ha constituido desde un enfoque de sostenibilidad débil y mantiene la preponderancia de los proveedores de capital, lo que ha desviado la intención original de promover un pensamiento integrado.

\section{VI.Conclusiones}

El incremento en la divulgación de información social y medioambiental por parte de empresas privadas y entidades públicas, reclama su contrastación con 
los débiles avances en la sostenibilidad de nuestra forma de producción y de interacción social. Por ello, la utilidad y la materialidad de este tipo de información, son dos de los temas de mayor debate en el campo de la contabilidad social y medioambiental en la actualidad (Unerman y Zappettini, 2014; GRI 2015a, 2016a; Torelli, Balluchi y Furlotti, 2020; Xiao y Shailer, 2021). Mucha de la información producida no atiende a las expectativas de amplios grupos de interés y el enfoque gerencialista de la divulgación adopta una postura que da preponderancia a los intereses financieros de los inversores o de las propias organizaciones. En este contexto, el stakeholder engagement se convierte en un camino para identificar e incorporar las expectativas de los grupos de interés en los procesos de gestión y de divulgación de la información de sostenibilidad, por parte de empresas privadas y de entidades públicas.

Este proceso va más allá de la participación de los grupos de interés en la producción de la información en la empresa, e implica el reconocimiento de sus expectativas y percepciones desde la elaboración de las guías y de los estándares. Ello es aún más relevante dado el interés de organismos emisores de normas de información financiera por participar en la estandarización de la información no financiera (IFRSF, 2020). De no incorporar dichas expectativas, el proceso de estandarización podría continuar dando prevalencia a los intereses de los inversores y de los mercados financieros, profundizando la banalización del accountability y la pérdida de utilidad y de materialidad de dicha información (O’Neill, 2002; Hood, 2010).

Dado que entidades domiciliadas en Colombia hacen parte de empresas líderes en la divulgación de información de sostenibilidad en Latinoamérica, en este trabajo caracterizamos las expectativas de diversos grupos de interés sobre dicha información. Según las percepciones de los encuestados, la información de sostenibilidad juega roles duales, tanto para la toma de decisiones como para la rendición de cuentas, pese a que prevalece la retórica de la utilidad de la información para las decisiones. Por ello, en el sector privado, los stakeholders consideran que los principales usuarios son los financiadores, los trabajadores y los auditores. Surgen posibilidades de temas que las guías y los estándares de mayor utilización en el contexto (GRI) no contienen de forma expresa. Por su parte, en el sector público se evidencian limitaciones en esta información, fruto de la extrapolación de temas del sector privado que se incorporan en las memorias de entidades públicas, también desde un enfoque de generación de valor financiero. Según las percepciones de los grupos de interés encuestados, los gestores públicos, los organismos de control y los planificadores públicos, serían los principales usuarios. Un gran reto es la generación de una información de sostenibilidad que resulte relevante para los ciudadanos, lo que implica ubicarlos como un grupo de interés central en el caso de las entidades públicas en sociedades democráticas. 
La utilidad de los informes de sostenibilidad en entidades públicas y privadas en Colombia...

En este contexto, la lógica de la estandarización podría ir en contravía de las expectativas de los stakeholders que parecen reclamar diferenciaciones entre la información de sostenibilidad de los sectores público y privado y, al interior de ellos, atendiendo a la variedad de contextos regionales, sectores y a la diversidad de conflictos socioambientales. Por todo ello, este trabajo contribuye en la caracterización y comprensión de algunas de las expectativas de diversos grupos de interés, desde una mirada alternativa a la planteada por el enfoque gerencialista de la sostenibilidad.

\section{Referencias Bibliográficas}

AccountAbility. (2008). AA1000 AccountAbility Principles Standard 2008. London. Recuperado de http://www.accountability.org/standards/

Almeida, B. A., Pinto, L. C., y Fonseca, A. (2021). Transparency of materiality analysis in GRI-based sustainability reports. Corporate Social Responsibility and Environmental Management, 28(2), 570-580. https://doi.org/10.1002/csr.2066

Amran, A., y Ooi, S. K. (2014). Sustainability reporting: Meeting stakeholder demands. Strategic Direction, 30(7), 38-41. https://doi.org/10.1108/SD-03-2014-0035

Argos S.A. (2018). Reporte integrado 2018. Recuperado de https://es.scribd.com/ document/413663440/Reporte-Integrado-Argos-2018

Argos S.A. (2019). Reporte Integrado 2019. Recuperado de https://argos.co/reporteintegrado/

Arias, J. P., Espitia, O., y Ruíz, F. (2016). La relación entre la Responsabilidad Social Empresarial y la Planeación Tributaria (Universidad de Manizales). Universidad de Manizales, Manizales.

Ariza-Buenaventura, D. (2012). El camino hacia la revelación: evolución de los informes de responsabilidad social en Colombia (2006-2009). Revista de la Facultad de Ciencias Económicas de la Universidad Militar Nueva Granada, 20(2), 97-120.

Bebbington, J., Brown, J., Frame, B., y Thomson, I. (2007). Theorizing engagement: the potential of a critical dialogic approach. Accounting, Auditing and Accountability Journal, 20(3), 356-381. https://doi.org/10.1108/09513570710748544

Bebbington, J., Larrinaga, C., y Moneva, J. M. (2008). Corporate social reporting and reputation risk management. Accounting, Auditing \& Accountability Journal, 21(3), 337-361. https://doi.org/10.1108/09513570810863932

Bortree, D. S., y Seltzer, T. (2009). Dialogic strategies and outcomes: An analysis of environmental advocacy groups' Facebook profiles. En Public Relations Review (Vol. 35). https://doi.org/10.1016/j.pubrev.2009.05.002

Cabrera-Narváez, A., y Quinche-Martín, F. L. (2021). Imag(in)ing Colombian post-conflict in corporate sustainability reports. Sustainability Accounting, Management and Policy Journal, 12(4), 846-871 https://doi.org/10.1108/SAMPJ-03-2019-0094

Cooper, S. M., y Owen, D. L. (2007). Corporate social reporting and stakeholder accountability: The missing link. Accounting, Organizations and Society, 32, 649-667. https://doi.org/10.1016/j.aos.2007.02.001 
Cruz, L. P., Vélez, C. A., y Romero, M. (2020). Beneficios tributarios por la adopción de políticas de responsabilidad social empresarial (RSE). Dictamen Libre, (26), 15-35. Recuperado de https://revistas.unilibre.edu.co/index.php/dictamenlibre/article/view/6168

Cunningham, P. H. (2011). State-Owned Enterprises: Pursuing Responsibility in Corporate Social Responsibility. Management Communication Quarterly, 25(4), 718-724. https:// doi.org/10.1177/0893318911419935

Deegan, C. (2020). The <IR $>$ Framework. An example of what unfortunately happens when people fail to comprehend the meaning of «accountability» take control of an important reporting initiative. En C. de Villiers, P.-C. Hsiao, y W. Maroun (Eds.), The Routledge Handbook of Integrated Reporting. New York: Routledge.

Devin, B. L., y Lane, A. B. (2014). Communicating Engagement in Corporate Social Responsibility: A Meta-Level Construal of Engagement. Journal of Public Relations Research, 26(5), 436-454. https://doi.org/10.1080/1062726X.2014.956104

Dumay, J. C., Guthrie, J., y Farneti, F. (2010). GRI Sustainability Reporting Guidelines for Public and Third Sector Organizations. Public Management Review, 12(4), 531-548. https://doi.org/10.1080/14719037.2010.496266

EJAtlas. (2019). Comunidad Wayuu, Guajira, resistiendo la desviación del Arroyo Bruno por la empresa El Cerrejón, Colombia. Recuperado 24 de abril de 2020, de https://ejatlas. org/conflict/glencore-switzerland-bhp-billiton-united-kingdom-angloamericanaustralia

Fasan, M., y Mio, C. (2017). Fostering Stakeholder Engagement: The Role of Materiality Disclosure in Integrated Reporting. Business Strategy and the Environment, 26(3), 288-305. https://doi.org/10.1002/bse.1917

Flórez-Parra, J. M., López-Pérez, M. V., y López-Hernández, A. M. (2017). Transparency and its determinants at Colombian universities. Higher Education Research \& Development, 36(4), 674-687. https://doi.org/10.1080/07294360.2016.1239613

Flower, J. (2020). The IIRC's journey: from sustainability to investor value. En C. de Villiers, P.-C. Hsiao, y W. Maroun (Eds.), The Routledge Handbook of Integrated Reporting. New york: Routledge. Recuperado de https://books.google.com.co/books?id=U7fjD $\mathrm{wAAQBAJ} \& \mathrm{pg}=\mathrm{PT} 4 \& \mathrm{lpg}=\mathrm{PT} 4 \& \mathrm{dq}=$ deegan + craig + integrated + reporting $\&$ source $=$ bl\&ots $=\mathrm{e} 0 \mathrm{sLZWqsWj \& sig}=$ ACfU3U1vU7QAhXqjUJiiO4FgO6DXsJ3PmA\&hl=es-4$19 \& \mathrm{sa}=\mathrm{X} \& \mathrm{ved}=2 \mathrm{ahUKEwjfjsuttqLqAhVlkeAKHUJkDIEQ6AEwAnoECAsQAQ \# v= \text {one }}$ page $\& q=$ deegan craig integrated reporting $\& f=$ false

Freeman, R. E. (1999). Divergent Stakeholder Theory. The Academy of Management Review, 24(2), 233-236. Recuperado de https://www.jstor.org/stable/pdf/259078.pdf

Freeman, R. E. (1984). Strategic management: A stakeholder approach (Vol. 1). Mashfield: Pitman. Recuperado de http:/www.mendeley.com/research/strategic-management-astakeholder-approach-2/

Friedman, M. (1970). A Friedman doctrine-The Social Responsibility of Business is to Increase its Profits. The New York Times Magazine, (13). https://doi.org/10.1007/9783-540-70818-6 14

García-Sánchez, M.I. (2021). Corporate social reporting and assurance: the state of the art. Spanish Accounting Review, 24(2), 241-269, https://doi.org/10.6018/rcsar.409441 
La utilidad de los informes de sostenibilidad en entidades públicas y privadas en Colombia...

Global Reporting Initiative. (2013). Sustainability Topics for Sectors: What do stakeholders want to know? Recuperado de https:/www.globalreporting.org/resourcelibrary/ sustainability-topics.pdf

Global Reporting Initiative. (2016). GRI Sustainability Reporting Standards. Recuperado 12 de febrero de 2018, de https://www.globalreporting.org/standards/

Global Reporting Initiative. (2019a). About GRI. Recuperado 21 de agosto de 2018, de https://www.globalreporting.org/information/about-gri/Pages/default.aspx

Global Reporting Initiative. (2019b). Reports List- GRI Database. Recuperado 2 de mayo de 2019, de https:/www.globalreporting.org/services/Analysis/Reports_List/Pages/ default.aspx

Gómez-Villegas, M. (2017). El Reporte Integrado en el Sector Público: Una mirada desde Latinoamérica. Revista Española de Control Externo, XiX(57), 67-92.

Gómez-Villegas, M., y Quintanilla, D. (2012). Los informes de responsabilidad social empresarial: su evolución y tendencias en el contexto internacional y colombiano. Cuadernos de Contabilidad, 13(32), 121-158.

Gray, R. (2007). Taking a Long View on What We Now Know About Social and Environmental Accountability and Reporting. Issues in Social and Environmental Accounting, 1(2), 169-198.

Gray, R. (2010). Is accounting for sustainability actually accounting for sustainability...and how would we know? An exploration of narratives of organisations and the planet. Accounting, Organizations and Society, 35, 47-62. https://doi.org/10.1080/096916 0X.2011.556420

Gray, R., y Collison, D. (2001). Can't See The Wood For The Trees, Can't See The Trees For The Numbers? Accounting Education, Sustainability and the Public Interest. Critical Perspectives on Accounting, 13(5), 797-836. Recuperado de http://boydquaich.standrews.ac.uk/media/csear/discussion-papers/CSEAR_dps-other-wood4trees.pdf

Greco, G., Sciulli, N., y D’Onza, G. (2015). The Influence of Stakeholder Engagement on Sustainability Reporting: Evidence from Italian local councils. Public Management Review, 17(4), 465-488. https://doi.org/10.1080/14719037.2013.798024

Greenwood, M. (2007). Stakeholder Engagement: Beyond the Myth of Corporate Responsibility. Journal of Business Ethics, 74, 315-327. https://doi.org/10.1007/ s10551-007-9509-y

GRI. (2014). G4 Sustainability Reporting Guidelines. Recuperado de Global Reporting Initiative website: https:/www.globalreporting.org/standards

GRI. (2015a). Defining Materiality: What Matters to Reporters and Investors. Recuperado de https://www.globalreporting.org/resourcelibrary/defining-materiality-what-matters-toreporters-and-investors.pdf

GRI. (2015b). Sustainability and Reporting Trends in 2025. Preparing for the future- First Analysis Paper. Recuperado de https:/www.globalreporting.org/resourcelibrary/ sustainability-and-reporting-trends-in-2025-1.pdf

GRI. (2015c). Sustainability and Reporting Trends in 2025. Preparing for the future- Second Analysis Paper. Recuperado de https:/www.globalreporting.org/resourcelibrary/ Sustainability-and-Reporting-Trends-in-2025-2.pdf 
GRI. (2016a). Defining What Matters. Do companies and investors agree on what is material?Mining, Metals and In collaboration with Electric Utilities. Recuperado de https:/www.globalreporting.org/resourcelibrary/GRI-DefiningMateriality2016.pdf

GRI. (2016b). The Next Era of Corporate Disclosure. Digital, Responsible, interactive. Recuperado de https:/www.globalreporting.org/resourcelibrary/The-Next-Era-ofCorporate-Disclosure.pdf

GRI, y UN Global Compact. (2017). Business Reporting on the SDGs: An Analysis of the Goals and Targets. Recuperado 8 de agosto de 2021, de https://www.unglobalcompact.org/ library/5361

GRI, UN Global Compact, y WBCSD. (2015). SDG Compass - A Guide for Business Action to Advance the Sustainable Development Goals. Recuperado 8 de agosto de 2021, de The SDG Compass website: https://sdgcompass.org/

Grupo Nutresa. (2018). Informe Integrado 2018. Recuperado de https://www.gruponutresa. com/wp-content/uploads/2017/03/Informe-Integrado-2016-grupo-nutresa.pdf

Grupo Nutresa. (2019). Informe Anual Integrado 2019. Recuperado de https:/grupobimbo. com/sites/default/files/Grupo-Bimbo-Informe-Anual-Integrado-2019_0.pdf\%0Ahttps:// webcorporativa.bankinter.com/www2/corporativa/es/inf_financiera_cnmv/informacion_ financiera/memoria/2019\%0Ahttp://www.grupobimbo.com/informe/Bimbo-Informe-

Grushina, S. V. (2017). Collaboration by Design: Stakeholder Engagement in GRI Sustainability Reporting Guidelines. Organization \& Environment, 30(4), 366-385. https://doi.org/10.1177/1086026616681612

Hood, C. (2007). What happens when transparency meets blame- avoidance? Public Management Review, 9(2), 191-210. https://doi.org/10.1080/14719030701340275

Hood, C. (2010). Accountability and Transparency: Siamese Twins, Matching Parts, Awkward Couple? West European Politics, 33(5), 989-1009. https://doi.org/10.1080 /01402382.2010.486122

Kolk, J. (2008). Sustainability, accountability and corporate governance: Exploring multinationals' reporting practices. Business Strategy and the Environment, 17(1), 1-15. https://doi.org/10.1002/bse.511

Manetti, G. (2011). The Quality of Stakeholder Engagement in Sustainability Reporting: Empirical Evidence and Critical Points. Corporate Social Responsibility and Environmental Management Corp. Soc. Responsib. Environ. Mgmt, 18, 110-122. https://doi.org/10.1002/csr.255

Mitchell, R., Agle, B., y Wood, D. (1997). Toward a Theory of Stakeholder identification and Salience: Defining the principle of who and what really counts. The Academy of Management Review, 22(4), 853-886.

Moratis, L., y Brandt, S. (2017). Corporate stakeholder responsiveness? Exploring the state and quality of GRI-based stakeholder engagement disclosures of European firms. Corporate Social Responsibility and Environmental Management, 24(4), 312-325. https://doi.org/10.1002/csr.1408

Mosangini, G. (2012). Decrecimiento y justicia Norte-Sur. Barcelona: Icaria.

Ngu, S. B., y Amran, A. (2018). Materiality disclosure in sustainability reporting: fostering stakeholder engagement. Strategic Direction, 34(5), 1-4. https://doi.org/10.1108/SD01-2018-0002 
La utilidad de los informes de sostenibilidad en entidades públicas y privadas en Colombia...

O’Dwyer, B., Unerman, J., y Hession, E. (2005). User needs in sustainability reporting: Perspectives of stakeholders in Ireland. European Accounting Review, 14(4), 759-787. https://doi.org/10.1080/09638180500104766

O’Neill, O. (2002). A Question of trust. Cambridge: Cambridge University Press.

O'Riordan, L., y Fairbrass, J. (2014). Managing CSR Stakeholder Engagement: A New Conceptual Framework. Journal of Business Ethics, 125, 121-145. https://doi. org/10.1007/s10551-013-1913-x

Portafolio. (2018). Diez colombianas están en índices de sostenibilidad. Recuperado 8 de febrero de 2019, de Empresas website: https:/www.portafolio.co/negocios/empresas/ diez-colombianas-estan-en-indices-de-sostenibilidad-521147

Rinaldi, L. (2013). Stakeholder engagement. En Integrated Reporting: Concepts and Cases that Redefine Corporate Accountability (pp. 95-109). Springer International Publishing. https://doi.org/10.1007/978-3-319-02168-3_6

Rodríguez-Bolívar, M., Garde-Sánchez, R., y López-Hernández, A. (2015). Managers as drivers of CSR in state-owned enterprises. Journal of Environmental Planning and Management, 58(5), 777-801. https://doi.org/10.1080/09640568.2014.892478

Rodríguez, G. A. (2016). Los conflictos ambientales en Colombia y su incidencia en los territorios indígenas. Bogotá, Colombia: Universidad del Rosario. https://doi. org/10.12804/tj9789587387407

Thomson, I., y Bebbington, J. (2005). Social and environmental reporting in the UK: a pedagogic evaluation. J. Bebbington / Critical Perspectives on Accounting, 16, 507533. https://doi.org/10.1016/j.cpa.2003.06.003

Thorne, L., Mahoney, L. S., y Manetti, G. (2014). Motivations for issuing standalone CSR reports: A survey of Canadian firms. Accounting, Auditing and Accountability Journal, 27(4), 686-714. https://doi.org/10.1108/AAAJ-07-2013-1393

Tilt, C. (2007). External stakeholders' perspectives on sustainability reporting. En J. Unerman, J. Bebbington, y B. O’Dwyer (Eds.), Sustainability, Accounting and Accountability (pp. 70-85). New York: Routledge.

Torelli, R., Balluchi, F., y Furlotti, K. (2020). The materiality assessment and stakeholder engagement: A content analysis of sustainability reports. Corporate Social Responsibility and Environmental Management, 27(2), 470-484. https://oi.org/10.1002/csr.1813

Unerman, J., y Zappettini, F. (2014). Incorporating Materiality Considerations into Analyses of Absence from Sustainability Reporting. Social and Environmental Accountability Journal, 0(3), 172-186. https://doi.org/10.1080/0969160X.2014.965262

Vaz Ogando, N., Ruiz Blanco, S., y Fernandez-Feijoo Souto, B. (2018). The assurance market of sustainability reports in Spain: An analysis from the demand perspective. Revista de Contabilidad-Spanish Accounting Review, 21(1), 48-62. https://doi.org/10.1016/j. rcsar.2017.07.003

Xiao,X., \& Shailer, G. (2021). Stakeholders' perceptions of factors affecting the credibility of sustainability reports, The British Accounting Review, https://doi.org/10.1016/j. bar.2021.101002 